\title{
Thinking may be more than computing*
}

\author{
PETER KUGEL \\ Boston College
}

\begin{abstract}
The uncomputable parts of thinking (if there are any) can be studied in much the same spirit that Turing (1950) suggested for the study of its computable parts. We can develop precise accounts of cognitive processes that, although they involve more than computing, can still be modelled on the machines we call 'computers'. In this paper, I want to suggest some ways that this might be done, using ideas from the mathematical theory of uncomputability (or Recursion Theory). And I want to suggest some uses to which the resulting models might be put. (The reader more interested in the models and their uses than the mathematics and its theorems, might want to skim or skip the mathematical parts.)
\end{abstract}

\section{Introduction}

It seems, to me, that parts of thinking may require more than computing. But that does not seem, to me, to mean that those parts cannot be studied scientifically. In this paper, I want to suggest some ways that the uncomputable parts of thinking might be studied with much the same precision, and in very much the same spirit, that Turing suggested for the study of its computable parts.

In his famous paper on "Computing machinery and intelligence" (Turing, 1950), Turing observed that one of the things that makes it difficult to study the relationship between thinking and computing is that, although we have a precise definition of "computing", we lack a precise definition of "thinking". Therefore, he suggested that, rather than try to study the whole of thinking (which we cannot define), we try to study specific parts of thinking (which we can point to). Let us, he proposed, look at specific activities that seem to

\footnotetext{
*I want to thank Peter Clote, Ben Cohen, Jim Gips, Peter Fejer, David Israel, Harvey Margolis, Ned Rosen and, particularly, the referees, for helpful suggestions. Correspondence and reprint requests should be addressed to Peter Kugel, Computer Science Department, Boston College, Chestnut Hill, MA 02167, U.S.A.
} 
require thinking and try to develop computable models for them, one at a time. When we succeed, the models we develop may tell us something about the parts of thinking that we have modelled.

I want to suggest that we might do much the same thing with the uncomputable parts of thinking. Let us look at parts of human thinking that seem (to some of us) to involve more than computing and try to develop precise uncomputable models of them. Insofar as our models are correct, they may tell us something about the uncomputable parts of thinking with much the same kind of precision that we now get from our computable models.

Turing's paper was addressed primarily to what we might think of as the engineering approach to the mind, in which we try to develop (or program) machines that do what the mind does. But his suggestion also applies to the scientific approach to the mind, in which we try to explain or predict what the mind does.

One reason that uncomputable accounts of the mind can be precise is that, like computable accounts, they can be represented by computer (but not by computing) programs. Computers can, in spite of what we call them in English, be used to do more than compute. (The fact that we call a machine a "computer" no more limits it to computing than the fact that we call a person "foolish" limits that person to foolishness.) Thinking may lie within the range of what a computer can do and still lie beyond the range of what it can do by computing alone. Turing suggested precisely this possibility in his 1950 paper. He devoted most of that paper to arguing that computers might be able to duplicate most of the behavior that, when people do it, we call "thinking". But, toward the end of his paper, he briefly suggested that they might also have to do more than computing to do it. Wrote Turing (1950):

Intelligent behaviour presumably consists in a departure from the completely disciplined behaviour involved in computation, but a rather slight one which does not give rise to random behaviour or pointless repetitive loops.

Cognitive Science now takes the first part of Turing's suggestion (that computers can model thinking) quite seriously. In this paper, I want to suggest that the time may have come to take the second part (that they will have to do more than to computing to do it) more seriously than we have been. The main aim of this paper is to suggest a way that this might be done systematically, using ideas from the mathematical theory of the uncomputable, or Recursion Theory. 


\section{How computers might do more than compute}

To see how a computer might do more than compute, consider an idealized general-purpose computing machine, $M$. When we use $M$, we first give it a program, $p$, and an input, inp. We then let it run, step by deterministic step, following the instructions of $p$, to process inp. We will refer to the process of $M$, running under program, $p$, on input, inp, as " $M_{p}($ inp $)$ ". From time to time, $M_{p}$ (inp) may print something. We will call anything it prints an "output". Since we will focus primarily on processes that make simple yes/no decisions, we will begin by limiting M's outputs to YES and NO. That is not as restrictive as it might, at first, appear and the generalization to more complex outputs is relatively straightforward.

We distinguish an output from a result. An output is anything $M$ prints, whereas a result is a selection, from among the things it prints, that we agree to pay attention to. This distinction is not important when we limit ourselves to computations because, when we use $M$ to compute, we agree to pay attention only to the first thing that it prints and often turn it off (or over to another job) once it has printed that. Thus its result and (only) output are one and the same.

Many problems can be solved by computations. Others can not. Among those that can not is:

The Full Halting Problem. Given a program, $p$, and an input, $i$, to determine whether or not $M_{p}(i)(M$, running under program, $p$, on input, $i$ ) will or will not halt (eventually).

A (totally) computable solution to this problem would be a computing procedure, controlled by a single program, $h$, such that $M_{h}(p, i)(M$, running under program $h$, on the input $(p, i))$ computes:

YES when $M_{p}(i)$ halts

NO when it does not.

There can be no such computing $M_{h}$ that produces the correct YES's and NO's for all possible input pairs $(p, i)$. However, a partially computable $M_{h}$, that produces only the YES's, is possible. (See the Appendix for an outline of the proofs.)

A different kind of procedure, that I will call (following Putnam, 1965) a total trial and error procedure, can produce both the YES's and the NO's. (The idea of a trial and error procedure was developed, apparently independently, by Putnam (1965) and Gold (1965). Precursors of the idea can be found in Shoenficld (1967), Popper (1959), in Leibnitz's (1956) writings about induction and in the work of Xenophanes (fragments 189 and 191 in Kirk and 
Raven, 1957).) The difference between a computing procedure and a trial and error procedure is this. When we run $M_{p}$ as a computing procedure, we count its first output as its result. When we run it as a trial and error procedure, we count its last output as its result. This makes a difference for, although no computing procedure can solve the Full Halting Problem, a total trial and error procedure can. For example, let $M_{h}(p, i)$ start off by printing NO. Then let it simulate (imitate) $M_{p}(i)$, step by step. If the simulation halts, let it print YES and halt. Count, as $M_{h}$ 's result, the last output that it prints. It is easy to see that this defines a trial and error procedure and that the result that $M_{h}$ produces, under this way of interpreting its outputs, is always a correct solution to the Full Halting Problem.

Notice that a machine running a trial and error procedure looks just like a machine running a computing procedure. It uses no special "hardware" and certainly no "magic". Its result is produced, like that of a computation, in finite time using only finitely much memory. But there is an important difference. When a computation (say to determine the value of $12+13$ ) comes up with a result (25), we know that that is the result. But when a trial and error procedure (say to determine whether Fermat's Last Theorem is true by checking, systematically, through all possible counterexamples) has output a YES, because it has not yet found a counterexample, we cannot be sure that that is its result. We only think that that is its result. It can always "change its mind". We know that its last output is correct but, at any given moment at which YES is its output, we do not know that YES will be its last output.

Trial and error procedures have been quite widely used to model the cognitive process of induction (Angluin \& Smith, 1983) and particularly grammatical induction (Osherson, Stob \& Weinstein, 1986; Pinker, 1979). They are one type of uncomputable procedure that we can run on a computer. But there are others, and other cognitive processes besides induction, that they can be used to model.

\section{3. "Geographies" of the mind and of the uncomputable}

I want to suggest several ways that parts of thinking might be modelled by uncomputable processes. To do this, I will pluck ideas from the mathematical theory of the uncomputable and set them down in various parts of the mind. To try to give some order to this process, let me skctch "gcographies" of the two territories involved - of the mind, where the ideas will sit, and of the theory of the uncomputable from which they will come.

There are various ways to divide up the mind, but one that suits me divides the mind into four parts, or modules (Figure 1): 
Figure 1. A "geography" of the mind.

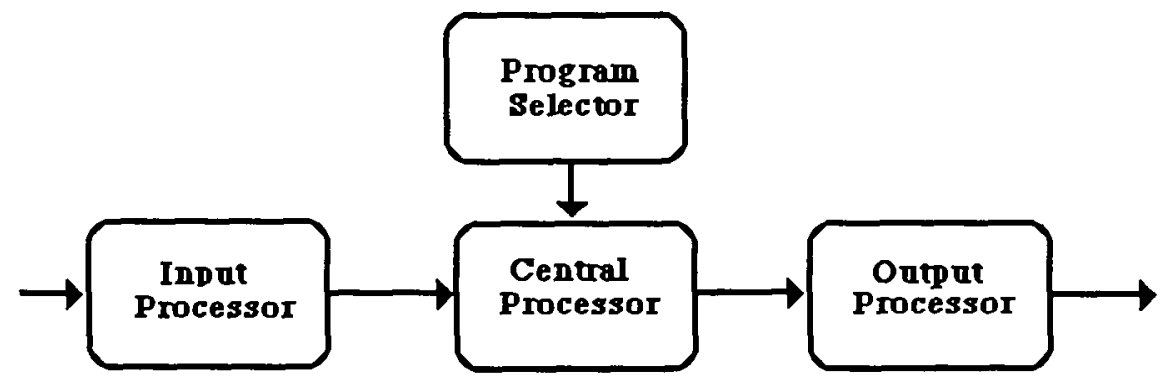

- An input processor that takes information from the sensors-say a pattern on the retina-and turns it into a form that the central processor can deal with. For example, it might take a visual signal and turn it into the message: THIS IS A SABRE-TOOTHED TIGER.

- A central processor that takes the results produced by the input processor and turns them into messages for the output processor. For example, it might take the message: THIS IS A SABRE-TOOTHED TIGER and turn it into the message: RUN.

- An output processor that takes the results of the central processor and turns them into something that can be used to affect the world. For example, it might take the message RUN and turn it into messages to control specific muscles to remove us from the immediate area.

- A program selector that selects a suitable program from the set of all available programs. It might, for example, study the situation and decide that it was time to use the ANIMAL RECOGNIZING PROGRAM rather than the BEAUTY APPRECIATION PROGRAM.

I want to suggest that each of these components might use procedures that require more than computing. But I want to say more than that. I want to say not just that these procedures are uncomputable but how they are uncomputable. Recursion Theory, the branch of mathematics that deals with the uncomputable, provides a theoretical framework for saying such things. In Recursion Theory, processes are not just uncomputable. Some processes are more uncomputable than others, or are uncomputable in different ways.

One way to divide up the territory of uncomputable processes is provided by the Arithmetic Hierarchy Theorem (Kleene, 1943; Mostowski, 1947). This hierarchy divides up the uncomputable processes in terms of the classes of predicates they can evaluate. At the lowest level of this hierarchy are the processes that can only evaluate totally computable predicates. A predicate, 
$P$, is said to be "totally computable", if there is a program, $p$, such that $M_{p}$ (inp) computes (produces, as its first and only output):

YES if $P($ inp $)$ is true.

NO otherwise

When we looked for a computable solution to the Full Halting Problem (above), we were looking for a totally computable solution. The class of all totally computable predicates is called " $\Sigma_{0}$ " (or " $\Pi_{0}$ "), and it forms the lowest level of the Arithmetic Hierarchy.

The Arithmetic Hierarchy Theorem tells us that, as we allow ourselves to use more and more quantifiers (the existential quantifiers ' $(\exists \ldots$...)' for 'There is a ... such that' and the universal quantifiers ' $(\forall \ldots)$ ' for 'For all ...') to define new predicates in terms of totally computable predicates, we can define predicates that are more and more uncomputable. For example, the predicate $S(p, i, t)$, that is true if $M_{p}(i)$ halts in exactly $t$ steps, is totally computable. But the predicate $H(p, i)$, definable in terms of $S$ by $H(p, i) \Leftrightarrow$ def $(\exists t) S(p, i, t)$, is not totally computable. (See the Appendix for a fuller discussion.) $H(p, i)$ is true if and only if $M_{p}(i)$ (eventually) halts and it expresses the Full Halting Problem. However, $H$ is partially computable in the sense that there is a program $h$ such that $M_{h}(p, i)$ computes:

YES if and only if $M_{p}(i)$ halts.

Note that only the YES is required. If $M_{p}(i)$ does not halt, then $M_{h}(p, i)$ need not print NO.

Predicates definable in terms of computable predicates, using at most one existential quantifier, fall into a class called $\Sigma_{1}$. In general:

$\Sigma_{n}$ is the class of all predicates definable in terms of totally computable predicates using, at most $n$ quantifiers, the first of which is existential.

$\Pi_{n}$ is the class of all predicates definable in terms of totally computable predicates using, at most, $n$ quantifiers, the first of which is universal.

( $\Delta_{n}$ is the class of all predicates definable in both ways using $n$ quantifiers, which is to say, of all predicates in both $\Sigma_{n}$ and $\Pi_{n}$.)

Arithmetic Hierarchy Theorem tells us that the sequence $\Sigma_{0}, \Sigma_{1}, \Sigma_{2}, \Sigma_{3}$, $\ldots$ is a proper hierarchy in the sense that $\Sigma_{n+1}$ contains all the predicates in $\Sigma_{n}$, together with some predicates that are more uncomputable than any in $\Sigma_{n}$ (Figure 2). And it says the same thing about the sequence $\Pi_{0}, \Pi_{1}, \Pi_{2}, \Pi_{3}$, $\ldots$, namely that $\Pi_{n+1}$ contains all the predicates in $\Pi_{n}$, together with some that are more uncomputable. It also says that, for all $n>0, \Sigma_{n}$ and $\Pi_{n}$ are distinct. Each contains predicates not in the othcr, but $\Sigma_{n+1}$ contains all the predicates in $\Pi_{n}$ and $\Pi_{n+1}$ contains all the predicates in $\Sigma_{n}$ (Figure 3 ). 
This is a rather "syntactic" way of defining these classes that does not seem to suggest much by way of models for the mind. But, for some of these classes, there are equivalent definitions that are more "semantic" and do suggest such models. For example, the lower $\Sigma$ classes can also be characterized as follows:

$\Sigma_{0}$ is the class of totally computable predicates, or predicates that can be evaluated by total computations, as we have already noted. (Our predicate $S$ was such a predicate.)

Figure 2. Relationships between the $\Sigma$ classes of the Arithmetic Hierarchy.

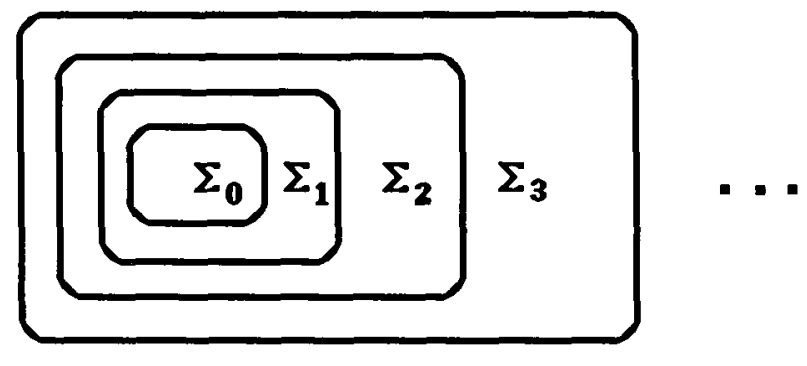

Figure 3. A "geography" of the uncomputable (a portion of the Arithmetic Hierarchy).

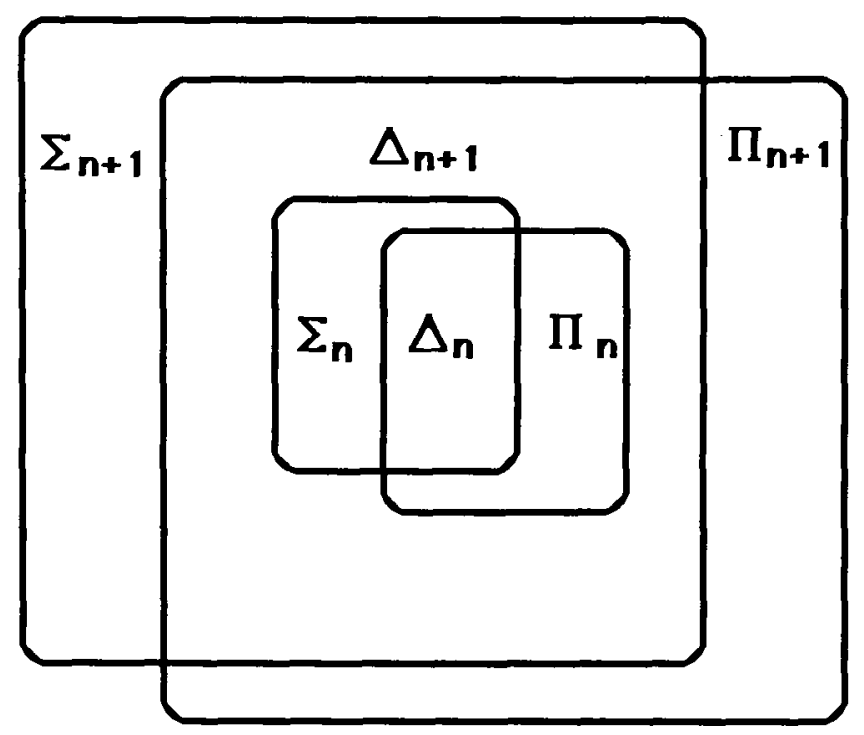


$\Sigma_{1}$ is the class of partially computable predicates, or predicates that can be evaluated by processes that compute YES when, and only when, the predicate is true, but do not necessarily compute NO when it is false. (Our predicate $H$ was such a predicate.)

$\Sigma_{2}$ is the class of (partial) trial and error predicates, or predicates that can be evaluated by processes whose YES results are their last outputs (rather than, as with computations, their first).

We generally consider the partially computable predicates (those in $\Sigma_{1}$ ) to be computable. So $\Pi_{1}$ is the lowest class in the Arithmetic Hierarchy that contains uncomputable predicates. Let's begin our search for uncomputable parts of thinking by looking in the input processor for features that might call for $\Pi_{1}$ models.

\section{The input processor and $\Pi_{1}$}

One thing that the input processor does is to classify what we observe. We see something and recognize it as a sabre-toothed tiger, as the letter A or as beautiful, and thus begin to have some idea of what to do with it. It has been suggested that our ability to classify objects as beautiful (Myhill, 1952), or as instances of the letter A (Hofstadter, 1982), might require more than computing. Myhill and Hofstadter both suggested that these abilities might involve the recognition of membership in productive sets. A set, $C$, is said to be "productive" (Post, 1944; Dekker, 1955) if no computing procedure can correctly recognize membership in $C$ and there is a computable function (called a "productive function") that effectively finds a counterexample to the claim that a given program, $p$, will computably recognize membership in $C$. Given a computing program, $p$, the productive function computes a member of $C$ that $p$ will not correctly classify. If we can recognize membership in a productive set, then clearly we can do something uncomputable.

The Arithmetic Hierarchy Theorem classifies predicates, such as the predicate $E(x)$ that is true of $x$ if and only if $x$ is an even number. It is not much of a generalization to use the same classification for sets, such as the set $E$ of all and only the even numbers. (We will sometimes usc the same letter for a predicate and the corresponding set.) The program, $e$, that computes the answer to the question $E(x)$ ? also computes the answer to the question "Is $x$ in the set, $E$, that is the range of the predicate $E$ ?", where the range of a predicate is the set of all objects for which it is true. In general, we will say that a set is in $\Sigma_{n}\left(\right.$ or $\Pi_{n}$ ) if it is the range of a predicate in $\Sigma_{n}$ (or $\Pi_{n}$ ).

There are productive sets in $\Pi_{1}$. An example of such a set is the set, $N$, 
of all pairs $(p, i)$ such that $M_{p}(i)$ fails to halt, or the set of all non-halting pairs. $N$ is the complement of $H$, the set of all halting pairs (the set of all pairs $(p, i)$ such that $M_{p}(i)$ halts). $H$ is partially computable since there is a partially computable procedure for determining membership in it. (Given $(p, i)$, simulate $M_{p}(i)$ and print YES if the simulation halts.) But there cannot be partially computable procedure for recognizing membership in its complement, $N$, and there is a productive function that computes exceptions for any computing procedure that is proposed as recognizing membership in $N$. (See the Appendix.)

There is, however, what we might call a non-halting procedure for recognizing membership in $N$. Consider the procedure, $n$, such that, if $M_{n}$ is given a prospective pair $(p, i)$ as input, $M_{n}$ prints YES and then simulates the operation of $M_{p}(i)$, step by step. If the simulation halts, $M_{n}$ "changes its mind", erases the YES and halts. We count $M_{n}$ 's last output (if there is one) as its result. (There is none if the YES is erased.) Clearly this procedure correctly identifies all and only the members of $N$ in the sense that it produces the result YES for all and only the members of $N$.

We can think of this procedure for identifying the non-halting pairs as a disproof procedure. It accepts a pair as a member of $C$ if it fails to prove membership in $C$. In contrast, we can think of a computing procedure as a proof procedure that accepts an object as a member of $C$ if it can prove membership. Notice that there is, in general, no moment at which a disproof procedure can do the equivalent of shouting Eureka! to announce that it has finally identified a member of $N$. The fact that its simulation of $M_{p}(i)$ has not yet stopped does not necessarily mean that it will not stop when we run it longer.

A computing procedure for recognizing members of a set can always be turned into an equivalent halting procedure, or a procedure that identifies an object as being in a set if and only if the procedure halts. (Simply have the procedure halt when it computes YES and, if it halts without printing YES, have it go into an infinite loop instead.) A non-halting procedure, on the other hand, identifies an object as being in a set if and only if it fails to halt. Membership in any $\Sigma_{1}$ set can be recognized by a halting procedure, membership in any $\Pi_{1}$ set can be recognized by a non-halting procedure. Membership in any totally computable $\left(\Sigma_{0}\right)$ set can be recognized by both kinds of procedures. (See the Appendix for a slightly fuller discussion.)

If the set of all occurrences of the letter A (for example) has the general "shape" of the uncomputable $\left(\Pi_{1}\right)$ set, $N$, then no computing (or halting) procedure can recognize all and only the A's. But a non-halting procedure could. It might take a pattern and "try" to prove that it is not an A. It would classify an input pattern as an $A$ if it failed in all its (possibly infinitely) many 
attempts at disproof. Such non-halting procedures are our first, and in some sense our simplest, examples of uncomputable models for cognition.

Just because something is done by a non-halting procedure does not necessarily put it beyond the range of computation. What one procedure does non-haltingly, another might be able to do haltingly (i.e. computably). For example, it is easy to define a procedure that fails to halt only for even numbers. But we know that the job of identifying members of this set can also be done by a computing procedure. However, non-halting procedures that are universal, in the sense that they can simulate all non-halting procedures, cannot be reduced to computations. This follows from the Arithmetic Hierarchy Theorem (see Appendix). If we could show that A-recognition was universal (or $\Pi_{1}$-complete), that would imply that it could not be reduced to computation.

Universality, in this sense, requires only the non-halting approach to getting results (which we are postulating for A-recognition) plus the ability to evaluate an arbitrary totally computable predicate under this approach. Our ability to determine which of the two "AorH's" in Figure 4 is an A seems, to me, to suggest that our A-recognizing ability has this additional ability.

Does the mind use a $\Pi_{1}$, or non-halting, procedure to recognize the letter A, or to do anything else? It's not easy to say. There cannot be a critical experiment, or observation, that would show that the mind does or does not use what we might call an "uncomputing procedure" to recognize A's. There is, for example, no instance of the letter $A$ that is so complex or difficult to recognize that it must be in an uncomputable set. What makes a set properly $\Pi_{1}$ (in $\Pi_{1}$ and not in $\Pi_{0}$ or $\Sigma_{0}$ ) is not its specific members, but the complexity of the borderline that divides its members from its non-members. Moving any one member, or even any finite number of members, from one side of this borderline to another does not change the level of the set's uncomputability in the Arithmetic Hierarchy. Thus, we might expect the observations that bear on whether the mind does or does not use processes that are productive, or in $\Pi_{1}$, (or both) to be global ones like the following:

- Mind changing. We do change our minds and computations cannot. (They can, by definition, produce only one result.) There can be little doubt that people occasionally put things into one classification only to later put it into another. We can characterize each such "putting" as a computation, but

Figure 4. Two familiar AorH's.

$$
\text { THE CHT }
$$


the whole process is more than a computation if one assumes that there is no final time at which we are sure the classification is finished. We may assume it is finished and act on it, but that does not prevent us from later changing it. Computation produces final results (We say that we know that $2+3=$ 5.) whereas cognition sometimes produces tentative results. (We say we think that some pattern is an A.)

- The short-falls of computable accounts. Hofstadter (1982) claims that our attempts to develop computational accounts of how we recognize (or generate) the letter A always seems to leave something out. There are computer programs that recognize A's in given type-fonts, A's that don't deviate too much from such type-fonts, and programs that may work well enough for many practical purposes. But there always seem to be some A's that are not included. (Hofstadter's article illustrates the problem with a nice collection of sample A's.) Similar observations have been made about the attempts to reduce other aspects of thinking to computations. The computing accounts often seem to fall short around the edges of the concept they are intended to account for.

This would not be surprising if the set of all A's were a productive set. We know that every productive set has a computable subset (see Theorem 6 in the Appendix). But we also know that such a computable subset is a proper subset-it leaves things out. Every time we characterize a computable subset of a productive set, we leave a part of the original set, which is also a productive set, uncharacterized. So the "left-overs" contain another computable subset. We can, in other words, keep approximating productive sets with computable ones. If the set of all A's is a productive set, we can get better and better computable characterizations of all of it. But, by the nature of a productive set, there must always be A's lcft out of any computable characterization, no matter how many times we improve it. Perhaps this is why so many of our attempts to give computable characterizations of cognitive processes continue to be improved but still continue to fall short of complete success.

- Recognition versus generation. We seem to be able to recognize, as beautiful, pieces of music that we almost certainly could not have composed. There is a theorem about the partially computable sets (see Theorem 5 in the Appendix) that says that there is a uniform procedure for turning a procedure for recognizing members of such sets into a procedure for generating them. Since this proccdurc is uniform-you can use the same one for all computable sets-it does not depend on any specific information about the set in question. So, if the set of all beautiful things were in $\Sigma_{1}$ (i.e. computable), we should be able to turn our ability to recognize beautiful things into one for generating them. (Such a general purpose ability would certainly be useful.) This 
suggests that a person who recognizes the Sistine Chapel Ceiling as beautiful, knows enough to paint it. Even though this claim applies to the knowledge involved, and not the painting skills, it still strikes me as somewhat implausible.

These arguments are, at best, only suggestive. That is probably all we can expect. The claim that the mind does more than compute is a claim about a (potentially) infinite model and our observations of human behavior, being necessarily finite, cannot fully constrain such models. Any finite behavior we observe can be modelled by a finite automaton, by an infinite Turing machine (limited to computing) or by a machine that does more than compute. At this level, our choice of one kind of model, rather than another, depends not on what it can account for, but on what it can account for smoothly. The arguments I have given suggest (to me at least) that non-computing machines might give us a smoother account of our observations than computing machines.

This is where Turing's proposal that we get more specific comes in. Since there seems to be no clearcut way to choose between computable and uncomputable models of thought, let us try to characterize specific aspects of thinking (such as A-recognition) more precisely, using one kind of model or the other. More specific models, by making more specific predictions, may be easier to test.

For example, the claim that A-recognition involves non-halting is already more specific than the claim that it involves more than computing, because we can go beyond computation by other than non-halting means. On the other hand, non-halting (which we can look for) need not imply more than computing because total computations can also be done by non-halting procedures (as we noted above). Notice how curious this becomes. Non-computing suggests non-halting but if we find non-halting, that does not prove we are doing non-computing. On the other hand, if we fail to find non-halting, that does not prove we only compute since there may be ways, other than nonhalting, that the mind might do more than compute.

There are deep philosophical problems about how a scientific paradigm, such as the non-halting paradigm, might be accepted or rejected, but they are (well) beyond the scope of this paper. Part of the decision probably depends on the results of observations. We might look at the results of reaction-time experiments. We might look for neurophysiological correlates. We might look for things that non-halting accounts explain better than halting ones.

The more specific features of our models (such as non-halting) can thus take on a life of their own. By developing more specific models, based on our general claim that the mind does more than compute, we can get more 
specific things to work with - much as the development of specific computable models has given Cognitive Science specific theories to work with. It is not my aim, in this paper, to develop any single uncomputable account in detail. But let me suggest how such accounts might be developed by filling in some more details in one possible account of A-recognition.

Let us begin by noting that A-recognizing procedures will tend to be used as parts of more complex procedures. The mind seldom attempts to answer questions, like "Is this an A?", outside the psychology lab. It usually tries to deal with more general questions like "What letter is this?" or, even "What is this?"

Non-halting procedures might be used in systems that attempt to answer such questions as follows. Suppose that the mind contains a family of nonhalting recognizers, say one or more assigned to each letter of the alphabet. Suppose, furthermore, that this family is controlled by a higher-level process (or parent) that, when it gets a letter to recognize, sends it to each member of its non-halting family (or child) for consideration. On receipt, each child tries to prove that the image it has been sent is not the letter for which it is responsible. When it succeeds, it turns itself off. If every child turns itself off, the parent process reports that the pattern is not a letter. If only one child fails to halt, then the parent chooses its letter as the answer. And if several children fail to halt, the parent process picks one of the surviving children's letter-perhaps, at this stage, using a computation to make its choice.

Unlike a parent, or controller, that chooses, perhaps in the manner of Selfridge's Pandemonium model (Selfridge, 1959), from among the halting, or computing, children, such a controller chooses from among the non-halting. If the mind worked in this way, we might expect some benefits including the following:

- Quick decisions. What Simon (1947) has called "satisficing"-finding a good, but not necessarily optimal, solution when we don't care to wait for the very best-comes naturally. If the parent process wants to, or has to, make a decision quickly, while several of its children are still going, it can choose (at random, if necessary) from among the not-yet-halted ones, knowing that at least some of the worst solutions have disposed of themselves by halting early.

- Negative decisions ("I don't recognize this.") can be made quite quickly: All sub-processes halt. Since one of the most interesting properties of what we observe is that so little of it is interesting, this capability could be useful. Most of what appears on our retina, for example, is not worth noticing. It could save the central processor a lot of work if the early stages of image processing filtered out the uninteresting-there being so much of it to filter out. 
- Positive decisions ("I do recognize this.") would signal themselves actively: A process that has not been disproved is doing something (still going) and thus there is no need for an added "Eureka!" signal that a halting subprocessor seems to need to signal success to its parent controller.

Benefits incurred by a proposed model of the mind, like the ones listed above, argue in its favor. Although the mind may not work in the best way possible, evolutionary pressures might lead it to "choose" good ways. Even if it turns out the mind does not use the better way, our work on it may not be wasted because we might be able to use its results in computer-based systems to do similar jobs.

The drawbacks of a particular model probably won't count in its favor as a candidate for use in building artificially intelligent machines, but they may count in its favor as a model of the mind we happen to be stuck with. If method $X$ can be expected to encounter difficulties of type $Y$, and we find that the mind encounters difficulties that look like $Y$, that could count as evidence in favor of the claim that the mind uses method $X$. At the same time, this could provide an "explanation" of why the mind encounters such difficulties.

Among the difficulties that might arise in the operation of the kind of pandemonic non-halting mind described above, that may have their parallels in human behavior, are the following:

- Stopping. A mind that contains a family of processes that fail to halt until they are disproved, might eventually have too many unhalted processes still running. These might expend energy, distract the main controller or otherwise degrade performance. Therefore, one might expect the mind to need procedures to stop unhalted processes. One way to do this is to have a "garbage disposal" unit that surveys the still running processes and manufáctures disproofs for them to fool them into halting. For example, if we have been running an "I want a candy bar" process all day, such a garbage disposal unit might manufacture an image of a candy bar to terminate (or disprove) that process, perhaps as we sleep. This suggests a starting place for an account of dreaming. There is more to dreaming than wish fullfilment, but it is a place to start. (It's where Freud started.)

- Going. Under certain circumstances, the halting, or garbage collecting, process might fail and a process might not halt. Such an unhaltable process (or family of processes) might then distract the controller (which, we assume, pays attention to unhalted processes), might set the garbage collecting units to vainly manufacturing disproofs, and otherwise confusing the situation. This suggests a beginning of an account of the mechanisms underlying neuroses. Again, there is more to neurosis than this account suggests, but it is a placc to start. 
- Switching. Sometimes, the controller might have to make a decision before all but one of its subsidiary processors had halted. Suppose, for example, in processing the phrase TAKE MY WIFE, it needs to pick a sense for TAKE. A natural one for it to pick in the sense in TAKE MY WIFE, FOR EXAMPLE. Suppose that this sense is then made implausible by the next word, as in comedian Henny Youngman's well known one-liner that then adds: ... PLEASE. The controller now has to drop the implausible sense and pick a new one, dropping its investment in the first and "listening" again to its unhalted processes. The mechanisms involved in such switching suggest an account of humor. It is a curious account for at least two reasons. For one thing, there is clearly more to humor than this, so it is, again, only a place to start. For another thing, it suggests that we have (in some sense) to have thought of a punchline before we hear it, in order to find it funny. That seems curious until we note that it also gives an account of why a joke does not really strike us as funny if we have to have it explained to us. This account predicts that people with attention deficits will lack a sense of humor, since they do not have much to shift. This "prediction" is supported by observation. (Levine \& Melmed, 1982).

- Practice. In our model, the parent controller might be a computing process that uses its unhalted children (when there aren't too many of them) as the data for its computations. Presumably, the more unhalted children it could consider in its computations, the better its results are likcly to be. Therefore, one might expect such a processor to practice ways of "hearing" as many of its unhalted children as possible. Certain human pastimes might serve this purpose-crossword puzzles and trivia games among others.

Such accounts should, like the other accounts suggested in this paper, be tested empirically before we take them too seriously. Some of them will almost certainly turn out to be wrong. Perhaps some may turn out to be, at least partially, right. Notice that they might help to bring, into the domain of Cognitive Science, some cognitive phenomena-such as humor, dreams, neuroses, play - that it has largely ignored.

Non-halting procedures suggest models for some kinds of remembering, including:

- Intermediate-term memory. The way we remember things for a day or two, only to forget them later, might be characterized in terms of non-halting procedures. Suppose, for example, that when we shave in the morning, we notice that we need razor blades. Imagine that this starts an 'I need razor blades' process going that seeks its own disproof. As we pass a razor blade display at the supermarket, later in the day, this still running process finds a prospect for a disproof in a razor blade display and it signals the central 
controller. We suddenly "remember" that we need razor blades.

This account suggests a second circumstance in which the controller might "hear" a sub-process or child. Perhaps it not only hears the last few (or only) survivors. Perhaps it sometimes also hears a child that has been running a long time, when it halts. Notice what has happened here. As we tried to apply our account, we found that we had to expand it. Thus observation can suggest extensions of our theory and there are various ways that the theory of when a parent process "hears" its children might be extended. Not all its extensions may come from observation. Some might come from other areas such as the theory of Operating Systems or the theory of Parallel Processes in Computer Science. Or extensions might come from a mathematical investigation of such processes.

- The Zeigarnik effect. It has been noticed (Zeigarnik, 1927) that we often remember unfinished tasks better than finished ones. While a waiter is waiting for a bill to be paid, he remembers the amount of the bill. Once the bill has been paid, the amount is forgotten. A non-halting account of this phenomenon might have the waiter's mind starting an "I need to be paid $\$ 34.56$ " process going, seeking disproof. The payment of the bill would serve as a disproof and halt the process. This is another side of intermediate memory.

- The stairway effect. There is other evidence that suggests that the mind uses non-halting processors. We have all had the experience of thinking of just the right thing to say, long after a conversation is over. That certainly looks like the work of a non-halting processor. If we flesh out an account of how this happens, we might get a testable account of one source of inspiration.

- The lineup effect. It scems to be quite casy to detcrmine when we do not know something or do not recognize something. We can look at a picture of a hundred people and see that there is nobody there we know. Witnesses who look over a police lineup, seem to be able to quickly determine that they have never seen the people in that lineup before. This seems easier to account for in terms of parallel non-halting processes than in terms of halting ones.

It also seems, to me, that it is easier to give accounts of how physical substances (drugs, hormones, alcohol) might affect the mind in terms of non-halting processes than it is in terms of halting ones. Consider, for example, the martini after work. Perhaps the day's work has started many nonhalting processes going. Activated, they vie for the attention of the parent processor whose process, though stronger than that of its children, gets confused by all the little processes asking for its attention. Alcohol (we hypothesize) quiets all processes down. The children become so quiet that 
many go unnoticed and the parent is happy, being less distracted. We feel better able to concentrate. Finally, with a few more martinis, even the parent is quieted down and we feel unable to concentrate at all.

Accounting for the cocktail hour is not high on the list of priorities for Cognitive Science and this account is likely to be wrong. (Even within the non-halting family framework, it is easy to think of other accounts.) But the mechanisms by which hormones and drugs affect the mind might succumb to similar accounts and such accounts, in terms of non-halting processes, might suggest mechanisms and cures. It seems easier to account for some relationships between mind and body in terms of non-halting accounts than in terms of halting ones.

One should not get carried away by all this (as, perhaps, I have been in the last few paragraphs). It is easy to find things that the theory one currently holds explains with panache. As Maslow once observed, to the person who has only a hammer, everything looks like a nail. To someone who has just hit on non-halting processes, they may seem to explain more than they probably do. One antidote to such exuberance is to try to use the resulting theories to predict what we do not already know. Experiments that test the predictions made by uncomputable theories, more thoroughly fleshed out, might help us decide what the mind does haltingly and what it does non-haltingly. I can think of no reason to think that the mind does everything haltingly or that it does everything non-haltingly. Probably, it does some things one way and some things the other. I am suggesting that we try to find out what is done how by developing theories (which $I$ have only hinted at above) and testing them experimentally (which I have not done at all).

\section{The output processor and $\Sigma_{2}$}

The mind uses what it knows-what it remembers of yesterday and what it perceives of today - to produce results it can use. Some of these results are used to do things-to ride bicycles, fry eggs, juggle tennis balls-that we do not yet know how to program computers to do. They seem to involve feedback, which resembles the idea of a non-halting process, but the difficulties we have in doing these things under computer control may be due to our difficulty in building the right kinds of transducers to turn information into actions. There are, however, several kinds of results that the mind produces that do not require transducers because they are limited to information processing and that still seem, to me and to others, to require more than computing. Among these are: 
- The general theories we derive from specific evidence.

- The specific facts we deduce from our changing knowledge of the world.

- The understanding we develop of the meanings of words and sentences that we hear.

There is a fairly extensive literature dealing with uncomputable accounts of the first and second of these. Uncomputable accounts of the way we develop general theories from specific evidence were suggested by Gold (1965, 1967). Subsequent work on this process of induction is the subject of a growing body of research summarized in Angluin and Smith (1983). (See also, Osherson, Weinstein, \& Stob, 1986.) Uncomputable accounts of how we deduce the implications of changing assumptions are implicit in several characterizations of non-monotonic reasoning. Logics that characterize such reasoning are discussed in many places, including the issue of Artificial Intelligence in which such articles as McCarthy (1980) and Reiter (1980) appear. Reiter (1978) seems to have been the first to note that such reasoning can involve more than computing.

Most accounts of these processes involve the trial and error procedures discussed earlier in this article. Trial and error procedures can do everything that computations can do, everything that the non-halting $\left(\Pi_{1}\right)$ processes of the preceding section can do, and more (see Appendix). Recall that a trial and error machine, or a computer carrying out a trial and error procedure, behaves exactly like a machine carrying out a computation. What differs is how we look for in its outputs. In a computation we take its first (and often only) output as its result. In a limiting computation, we take its last output as its result.

Suppose, for example, that we are trying to find the pattern that underlies the infinite sequence:

$$
01010101010101010101010101010101 \ldots
$$

We can think of the process of doing this as going from an infinite sequence, of which we can only see a finite portion at any time, to a finite program that generates the sequence. Such a program would give us at least some of what we want of a theory of such a sequence-the ability to predict its members. To predict, we run the program. And it would reduce the infinite data of the sequence to a finite object that we can store in the finite space between our ears.

If the process by which we generate this theory from evidence is a computation, then there must come some time in its processing that it produces its result. For example, it might conclude, say after seeing 100 symbols, that the 
full sequence consists of 01 repeated indefinitely. Since we are treating it as a computation, we cannot count any later outputs as its result ... even if it should turn out that after, say, 200 symbols, the sequence tails off into all 0 's. If, however, we are treating the process as a trial and error procedure, it may change its mind when such contrary evidence comes in. The first output may, but need not, be its result. Trial and error procedures have to settle on a final result eventually or else their result is undefined. But they do not have to tell us when they have done so. They are not the kind of procedures one wants to use in a computer center, but their behavior seems, to many, to be a better model of the way people derive theories. It is not intelligent to stick to a theory as countervailing evidence builds up (although people do sometimes do it), no matter how well founded the theory may have once appeared.

Gold (1965) suggested this sort of process as a model of what scientists do when they come up with finite theories of potentially infinite sets of evidence. It is a version of Popper's (1955) account of the scientific method, according to which science gets its results, not once and for all, but by something like a trial and error process. Popper speaks of science as having, not an effective (or computable) proof procedure, but an effective disproof procedure. That is another way of characterizing trial and error procedures.

The computable account of the scientific method-according to which science has an effective proof proccdure-is what we might think of as the "Eureka!" account. This account is embodied in the stories of Archimedes in the Greek bath, Newton under the English apple tree and Einstein on the Swiss trolley. It is also the first account of scientific method that most of us encounter in school. But, in spite of its intelligent exemplars and its early appearance in our intellectual lives, the computable way of deriving finite truths (or theories) about an infinite world, may not be particularly intelligent. True, there are good reasons for preferring the computable way of deriving knowledge. We know the results of computations and only think we know the results of trial and error procedures. There are many reasons for preferring knowing to thinking (as Popper, 1966, observed). But that does not change the fact that sometimes thinking may be more appropriate.

Trial and error procedures can evaluate all the predicates in $\Sigma_{2}$ of the Arithmetic Hierarchy, and, hence, also all the predicates in $\Pi_{1}$. The procedures that follow the Gold model to derive theories from evidence, differ in several ways from the procedure discussed in the previous section, that recognizes A's. For example, where the input of the A-recognizing procedure was a finite pattern, the inputs of our theory-deriving procedures are infinite. And the outputs, rather than coming from a set of two things (YES or NO), can now come from an infinity of things (e.g. the set of all possible programs). 
We are, in other words, now dealing with procedures to evaluate what mathematicians call functionals, whose ranges are the integers. (A brief discussion of what is involved in switching from predicates to such functionals appears in the Appendix.)

There are various activities of the mind that might be modelled by procedures for evaluating trial and error functionals, but to fix ideas, let's look at how they might be used in a specific process-the process by which we deal meaningfully with words and sentences in natural languages. Again, there are some general considerations that suggest that our dealings with meanings might involve trial and error procedures. The tentativeness of their results, and other global properties of trial and error procedures, seem to be reflected in observable features of the process by which we understand the meanings of utterances. For example:

- Mind changing. We do change our minds about the meanings of specific utterances (or word tokens) and the number of times we are able to change our minds about such meanings does not seem to have an a priori limit. (Our mortality does enforce a limit, but we tend to ignore such things in abstract theories-much as we ignore the limit that (say) the size of the universe places on a computer's memory when we represent computing abstractly in terms of Turing machines.) Consider the word BOW in THIS IS A BOW. In this sentence it can have several meanings. We seem to select one when we hear the word. Perhaps we choose the kind of bow we tie on presents. When we hear it in a broader context, as in THIS IS A CURTSY AND THIS IS A BOW, we (probably) choose another sense. But this change is not "permanent" because we can revert to the original if we add more context as in: THIS IS A CURTSY AND THIS IS A BOW. TIE IT IN YOUR HAIR BEFORE YOU CURTSY. The meanings of words can be changed by other words and/or events, or even just more thought. What changes them can be arbitrarily far away from what is changed. Thus something that happens at (say) the end of a detective story, can change the meaning of what was said near the beginning.

- Learning. We also change our minds about the meanings of words in general (or word types). Consider, for examples, how the word FORCE changes its meaning to a student taking an introductory physics course. Meanings seem to behave, at least in some respects, like theories in the Popper/ Gold trial and error account of scientific method.

- Recognizing versus generating. We seem to be able to understand what we cannot generate. We can understand words we cannot use properly, we can understand a novel that we cannot write and more. This argues for an uncomputable (not necessarily trial and error) account of understanding in 
much the same way that our ability to recognize beautiful things we cannot generate argues for the uncomputability of the set of all beautiful things.

- Short-falls of computable theories. Our attempts to develop computable accounts of what we do when we understand, seem to suffer from the same kind of "we are not quite there yet" problems as our attempts to develop computable accounts of how we recognize the letter A. Perhaps there are productive trial and error sets involved.

These features of our semantic behavior suggest that uncomputable (possibly trial and error) processes might be involved in our handling of meanings. But, again, we need to flesh out our accounts of how we handle meanings uncomputably before we can say much more. There are many theories of what meanings are, and probably uncomputable versions of most. To fix ideas, let's settle on an account that seems to me to be rather neutral. Let us say that the meaning of a word, such as FISH, is a predicate, $F$, that is true of those situations in which the word FISH is correctly uttered and false of all others. The ability to compute such meaning predicates could account for some of our ability to deal meaningfully with words, much as our ability to compute the prediction predicates, that represent sequences in Gold's account of induction, could account for our ability to use theories to predict. A meaning predicate (like $F$ ) might be represented in the mind by a program, $f$, that the mind can apply to an internal representation of a situation to produce a judgement of whether or not the word, whose meaning $f$ represents, could be properly used in that situation.

In a theory of semantics, based on such meaning predicates, some things that we do with meanings might be represented by trial and error (rather than computing) procedures, including:

- Acquiring them. Gold (1967) suggested trial and error accounts of how we acquire the "syntax predicates" (or grammars) of our native languages. We might develop trial and error accounts of how we acquire "meaning predicates" (like $F$ ) too. It hardly seems plausible that a child learns the meaning of (say) FISH I computably. The meaning of FISH seems to change, for a given child, over time. It grows as a child's acquaintance with fishes grows. At first, it might only cover the living-room goldfish. In time, it might spread out to cover eels and salmon steaks. It might shrink when the child learns that a whale is not a fish. And it might be refined if the child becomes a tropical fish fancier or an ichthyologist. The predicate $F$, as understood by a given person, seems to become true where it used to be false and false where it used to be true. The functional $L$, that learns this predicate $F$, thus seems to behave like a trial and error functional. Ask yourself: "When do we have the "Eureka!" moment at which we finally discover the final and total 
meaning of a word like FISH?' The fact that we don't appear to always have such a moment can be accounted for by a trial and error account of the process of meaning acquisition. A full theory of this predicate-acquiring process would, of course, have to distinguish errors made in applying $F$ (That looks like a fish but it's only an old shoe), which we are ignoring, from errors due to an incorrect internalized definition of $F$. (That whale is what I think of as a fish because I don't (yet) know that a whale is not a fish.)

- Verifying them. Even if we assume that we have acquired the correct meaning predicate for FISH, the process of verifying the truth of THERE IS A FISH IN THAT POND, might still be a trial and error process. (That certainly looked like a fish.) If trial and error processes are involved in both acquiring the meanings of words and in determining whether the utterances that use those words are true, then the combined process of acquisition and verification could be more uncomputable than either alone (see Appendix). Thus it should not surprise us when issues that involve both processes are difficult to resolve.

- Using them quickly. The mind seems to manage with "hardware" that is slow by the standards of the modern computer. One advantage of the mode of organization that we have been discussing - a family of disproving sub-processes run by a computing parent-is that it might speed up processing by allowing a high degree of parallelism, without communication between subprocesses. Suppose, for example, that we recognize that something is a FISH, or a LETTER, by starting a whole family of processers, each of which has its own procedure for giving a negative answer and "going to sleep". This could speed up both recognition (the parent waits until only one or a few of its children stop) or non-recognition (all children stop). By this account, both recognition and non-recognition should take approximately the same amount of time. This is not what one would expect if such recognition/non-recognition were done serially, by checking through each possibility in turn. In serial processing, non-recognition (which requires examining all the possibilities separately) should take longer than recognition (which could stop at the first success). This suggests one account for an observation of Sternberg (1966): Subjects asked to determine whether a given digit falls into a small reference set of digits, take about as long to determine that a probe digit is in the set as to determine that it is not in a set.

- Using them to choose what to say. One of the best signs that a person understands a language is his or her ability to speak it, and speaking involves knowing what to say and when. The fact that we edit our own writings suggests that choosing the right word might involve a trial and error process rather than a (one shot) computation.

Choosing what to say can also involve an uncomputable process in another 
way. Suppose we want to convince someone to do something. If the accomplishment of that thing can be recognized computably, then choosing what to say might be represented by a computation. (We keep generating utterances until our goal has been accomplished.) But in many cases we cannot tell, with finality, when we have accomplished our goal. Suppose, for example, that we are trying to explain something. How can we tell when we have succeeded? Usually, we can't because there is no easy computable test of whether or not something is (really) understood. We may ask questions on tests, the answers to which may make us think we have succeeded, only to find later that we have not and need to explain some more. Teaching, which is a kind of communication, seems, at times at least, to be a trial and error process of roughly this kind.

- Using them to understand utterances. Even if the meaning of FISH is represented by a computable predicate, $F$, the process of using $F$ to understand an utterance of the word FISH might involve more than computing. Consider the following theory of what it means to understand an utterance of a word. We hear a sentence such as THERE IS A FISH IN THAT LAKE as we stand next to a lake. Understanding that utterance of FISH involves completing a description of the situation in which it is uttered so that the utterance becomes correct. In this case, for example, we might fill it in by postulating a large bass in the lake. But we may be wrong (or the speaker might have been mistaken, lying or what have you). Perhaps the speaker only meant to say that there was a minnow in the lake, which, if true, would not have gotten us to go fishing. A full explication of such an account is well beyond the scope of this paper but, if such an account is correct, the process of completing the partially specified situation might be represented as the process of inverting the predicate $F$. Given that person $U$ uttered FISH in situation $S$, fill in the specification of $S$ so that the utterance becomes appropriate. (A discussion of such inversion processes and how they might involve trial and error procedures appears in the next section.)

- Combining them. We seldom use words in isolation. We combine words to make phrases, phrases to make sentences and sentences to make discourses. We assume that, in most cases, the meanings of the compound utterances can be derived from the meanings of their components in relatively uniform ways. And we are suprised when this does not happen in the way we expect.

For example, consider the ways we judge the probability of utterances being true. Tversky and Kahneman (1983) asked subjects to judge how likely it was that a woman described as a "social activist" was (a) a bank teller and (b) a bank teller who was active in the feminist movement. Even among those familiar with probability theory, more said (b) was more likely to be true 
than (a), in spite of he fact that (b) logically implies (a) and hence, by the standard account of joint probabilities, cannot be more likely. But suppose that we judge likelihood by trying to disprove unlikelihood. So we judge the likelihood of the woman's being (a) or (b) not by judging that, but by trying to disprove that she is not (a) or (b). To make such a judgement, we start two processes:

(disprove a): Try to show she's not a bank teller.

(disprove b): Try to show she's not a bank teller and a social activist.

Each process goes its merry way, but disproving (b) runs into more problems than disproving (a) and so it gives a weaker response. Conclusion: We take (a) to be more "likely".

Or consider the problem of trying to account for the way we choose paradigms for compound noun phrases in terms of the way we choose paradigms for their components. 'The goldfish, which is not a good paradigm for either PET or FISH, seems, to most people, quite a good paradigm for the phrase PET FISH. How does it get there? Suppose we identify the meaning of a noun with a set of paradigms, or frames that represent the features of such paradigms, as suggested by Rosch (1978). Imagine the parent of such a family of paradigms, sending a noun to its children for consideration. Suppose that the children become "quieter" as they find partial disproofs of their paradigm. Let the parent choose the "loudest". In the processing of a noun phrase, several such families might be involved. Look first at the PET family. At any moment, it may have several children "still going and not yet fully disproved". The parent chooses the "loudest" temporarily, but when it hears from another family that refuses to accept it, it may have to choose another. For example, suppose that the PET family has picked a cocker spaniel as its paradigm. The family dealing with the next word, FISH, refuses to accept this paradigm and so the PET family rejects that child and goes further down its list of not yet disproved paradigms to find an acceptable partner for PET, settling for the relatively quiet (but still "loudest") goldfish child. There are various ways we might develop an account of how such families communicate that are consistent with the findings of Smith and Osherson (1984) about the combination of paradigms.

The combination of word meanings into phrases offers a rich source of researchable problems for various reasons. From the psychologist's point of view, the words combined provide an easy variable to manipulate in experiments. For the mathematician, operations on a set of objects are easy to study, using ideas from algebra and logic.

There are many ways that accounts of how families of non-halting processes interact might be developed. And there are many different ways we 
might account for semantic phenomena. The computability or uncomputability of an account, alone, would not, it seems to me, determine whether it is right or wrong. What determines that is what we observe and that, in turn, depends on what nature has chosen to do inside our heads. And the way to find that out is to develop theories (both computable and uncomputable) and, as Popper suggested, try to disprove them. I have only sketched some ideas for uncomputable accounts of semantic phenomena. These sketches, and theories based on alternative ideas, need to be developed in more detail before we can even begin to ask if they are correct. One way to work on uncomputable "familial" accounts is to spell out clear rules about how "parents" choose "children" and then how such "families" interact. With these rules clearly specified, we can test their predictions against observations.

Trial and error accounts of cognitive processes need not, of course, be limited to the areas that I have discussed in this section. They have been proposed (Aizerman et al., 1964) for pattern recognition and they could undoubtedly be used in other areas as well. We might take any one of a number of existing theories and look at them "through trial and error glasses". When looked at in this way, they might lead to more fluid, more dynamic, accounts of thinking.

\section{The central processor and $n$-trial procedures}

It has been argued that the mind can do more than compute because it has access to special kinds of "hardware" in its central processor-perhaps random (rather than deterministic) elements or analog (rather than digital) ones. One runs into problems when one tries to make such (intuitively appealing) suggestions precise, particularly when one tries to do it in the kind of framework that I have been using, which ignores matters of efficiency. For example, it is not easy to say what a random element could add, within this framework, that could not be achieved computationally, using a pseudo-random number generator. Analog elements might increase efficiency, but it is hard to see what else they would add that could not be done with digital approximations (but see Appendix for a brief discussion of some possibilities in both these directions).

Whether or not we can work out such "hardware" accounts of how the central processor might do more than compute, there are accounts of how the central processor might go beyond computing, using only the deterministic, digital "hardware" of the computer, that focus on how we interpret the behavior of such deterministic digital machinery. For example, McCarthy (1956) has suggested that the process of problem solving might be represented by the: 
Problem of inverting computable functions. Given a program, $p$, and an output, $o$, find an input, $i$, such that $M_{p}(i)$ computes $o$.

The problem of inverting computable functions differs from the problems of evaluating and inducing computable functions. When we are asked to evaluate a computable function, we are given the program and an input, and asked to find the output, as in $2+3=$ ?. When we induce such a function, we are given the inputs and outputs and asked to find the function, as in 2 ? $3=5$. When we invert, we are given the program and an output, and asked to find an input that would produce the required output, as in ? $+?=5$. Computable procedures are, obviously, well suited for dealing with problems of the first kind, but that need not (and, I believe, does not) suit them particularly well for dealing with the other two types of problems.

Take inversion. Some kinds of problem solving can be represented, as McCarthy suggested, by the process of inverting computable functions. Consider, for example, the following problem: Find two integers, that can be expressed in decimal notation without using zeros, whose product is exactly $1,000,000$. Here we can think of ourselves as being given a program that checks a potential answer for correctness. Given two candidate integers, it computes YES if they solve the problem and NO if they do not. Solving the original problem is then equivalent to finding an input pair-there is only one in this case-that, when input to this program, produces the output YES.

McCarthy (1956) gave some reasons for thinking that all well defined problems may be representable in this form. He also noted that there is always a partially computable solution to the problem of inverting computable functions: Try all the possible inputs, one after the other, until you find one that produces the required output. To handle the fact that some inputs may cause a program to produce no outputs at all, we can "dovetail" the individual computations, doing the first one for one step, the first two for two steps and continuing to do the first $n$ for $n$ steps, as $n$ grows. At each step, we throw out any computation that halts unless it has computed the sought-for output. If that happens, print the input(s) as the result and stop. (This solution is not totally computable because it does not always tell us when no solution is possible. However, it is partially computable because it will compute a solution in all the solvable cases.)

But the process of inverting computable functions really characterizes puzzle solving better than problem solving. In a puzzle, the best solution can be recognized effectively, once it has been found. This is not always the case with problems. A problem is more like asking: "What is the best way to join two picces of wood (for somc specific purpose)?" Given a problem, we may not 
be able to tell, effectively, when a "best" solution has been found. There are many ways to join two pieces of wood: nails, glue, clamps, screws, and so forth. We want the best one but, when we find it, we may not know that it is the best possible. There may be better ways that we haven't considered yet. Therefore, solving this kind of problem is probably better represented by:

The problem of approximately inverting computable functions. Given a program, $p$, a desired output, $o$, and a measure of the "distance" between two outputs, find an input, $i$, such that the result computed by $M_{p}(i)$ is as "near" as possible to the desired output, $o$.

This problem comes in two "flavors", that lie at slightly different levels of uncomputability. If we allow our distance measure to take arbitrary real numbers as values, then, no matter what non-zero distance we get, there is always a chance of improvement. Under this condition, the problem of approximately inverting computable functions becomes a problem whose general solution requires a trial and error procedure. It tries all possible solutions, outputting, at each stage, the "best" solution so far. Of course, there may be no best because better and better ones keep arising.

The situation is slightly different when we allow only integer distances. Then, when we find a solution at a distance of (say) 6 from the ideal, we know that we can only find six better solutions (at distances of 5, 4, 3, 2, 1 and finally 0 ) and hence that we will need to "change our mind" at most six times. Procedures that can "change their minds" at most $n$ times have been called " $n$-trial procedures" (Putnam, 1965). A 1-trial procedure is a partially computing procedure and, for any $n$, there is always an $(n+1)$-trial procedure that can do more than any $n$-trial procedure can (see Appendix). Thus the $n$ trial procedures never do all that trial and error procedures can do. There is an extensive theory that characterizes this territory (see, for example, Epstein, Hass \& Kramer, 1981; Posner, 1980).

The mind might use $n$-trial procedures to solve problems. It might use them to learn concepts from examples by allowing itself to "change its mind" at most $n$ times before settling on its final concept. (After all, it can't spend all its time learning. Eventually, it has to start doing.) Or it might use them to choose paradigms as "meanings" of words if there are only $n$ paradigms to be considered and none is considered more than once. In each of these cases, the size of $n$ would be one measure of the mind's ability to adapt to its environment. All other things being equal, the higher $n$, the more finely tuned the final problem solution, the final concept learned, or the meaning assigned, could be. On the other hand, the higher $n$ was, the longer the 
processes of problem solving, learning or understanding might take.

Characterizing problem solving in terms of $n$-trial processes suggests some ways that we might go about studying this process scientifically. For instance, we might ask:

- Is there a single $\mathrm{n}$ for all minds? There seem to be several universal constants that govern the mind, such as Miller's (1956) seven-plus-or-minustwo estimate of the size of short-term memory. There may be a single $n$ for all human minds or there may be different ones for different minds. $n$ is one measure of a system's learning/problem-solving/understanding ability and it would be interesting to know if all people had the same one. (There may be different (smaller) $n$ 's for animals. A problem solution derived by a 0 -trial procedure would be innate.)

- If different minds have different $\mathrm{n}$ 's, can the $\mathrm{n}$ of an individual mind be measured? If human minds have different n's, there may be some tasks for which high $n$ 's are better than low ones and vice versa. Attempts to measure $n$ in individuals (if their $n$ 's can be different) might prove interesting. Success might also prove useful because knowing an individual's $n$ might help us to better teach that individual and/or fit him or her to tasks. (Presumably some tasks would be better done by those who spend a lot of time learning and others would be better done by those who get right down to doing.)

- Do different parts of a single mind have different $\mathrm{n}$ 's? If they did, the ability to measure them individually might give us an interesting multi-dimensional assessment of a human mind that could be used to do something to improve that mind. This suggests another question.

- Can we intervene to change $\mathrm{n}$ ? IQ tests today are usually used to evaluate and classify students in schools. But suppose that such tests could be made diagnostic so that one might get a result like: "This student has a rather low $n$ in dimension $X$." One might then use that information to try to raise that $n$ rather than just living with it-as we now seem willing to live with a student's measured IQ.

- Does it make sense to try to control $\mathrm{n}$ in artificial problem solving systems? For example, a computer system, doing medical diagnosis, might be thought of as a problem solving system, attempting to approximately invert computable functions. It is given a set of rules (or a program implementing those rules) that describes how the body works, together with a set of symptoms (or outputs of that program) and asked to find an input or inputs (diseases, poisons and the like) that might have produced the given outputs. Controlling the number of times it was allowed to "change its mind" might let us control the speed/accuracy tradeoffs for such a system to fit it to our purposes. 
Measuring and controlling $n$ might help us intervene to change human intellectual performance and to control the performance of "thinking" machines. If such interventions work, that would count as evidence in favor of the claim that such $n$ 's exist. We tend to accept theories that successfully resist disproof. We admire theories that predict. But we use theories that work. Working well is, thus, evidence in favor of a theory. After all, if a theory works, we may not care whether or not it is also correct. (This might be important in choosing uncomputable theories since they seem to resist some of the more direct ways of determining whether or not they are correct. See the discussion in Section 8, below.)

The processes of inverting, and approximately inverting, computable functions might serve as precise models to clarify some philosophical proposals. For example, Peirce suggested a third form of inference (in addition to the traditional deduction and induction) that he called abduction. He gave several different characterizations of abduction, but one that is particularly easy to understand was given in terms of the syllogism (see Peirce, 1878, and Figure 5).

What Peirce called a "rule" is a general principle that is applied to specific examples, much as a program represents a general principle that a computer applies to specific inputs. What Peirce called a "case" is what the rule is applied to, much as a computer's program is applied to its input. And what Peirce called a "result" plays a role not unlike that of a computcr's output. This analogy suggests that we might model deduction as what I called "evaluation" above, which is often done today. We often think of axiomatic theories as recursively enumerable (or partially computable) sets of theorems. It suggests that we might model induction by what I called "induction" above, following Gold $(1965,1967)$. And it suggests that we might model abduction by inversion. Such a model, perhaps along the lines of the model of medical diagnosis suggested a moment ago, might help clarify Peirce's ideas about abduction.

The theory of the uncomputable suggests a number of precise models for philosophical ideas, and such models might help clarify them. Some of the things that philosophers, such as Dreyfus (1979), have suggested that "com-

Figure 5. Three types of inference (Peirce, 1878).

DEDUCTION

Rule: All men are mortal. Case: Socrates is a man. Result: Socrates is mortal.
INDUCTION

Case: Socrates is a man. Besult: Socrates is mortal. Rule: All men are mortal.
ABDUCTION

Rule: All men are mortal.

Besult: Socrates is mortal. Case: Socrates is a man. 
puters cannot do", might find formal models in Recursion Theory. Several philosophical problems associated with induction might be clarified in terms of trial and error models (Kugel, 1977). Uncomputable models of philosophical proposals might increase their precision, a merit that should not be taken lightly.

In psychological models of the mind, $n$-trial procedures might play roles outside the central processor. For example, the problem of deciding 'What lower-case letter is this?' might be suitably represented as a 26-trial process for our 26-letter alphabet. A variant of the class of $n$-trial procedures that sits between $\Pi_{1}$ and $\Pi_{2}$, much as the class of $n$-trial procedures sits between $\Sigma_{1}$ and $\Sigma_{2}$, might characterize the kinds of processes that we discussed in the previous section where, instead of choosing between halting $\left(\Sigma_{1}\right)$ alternatives, we choose among non-halting $\left(\Pi_{1}\right)$ alternatives. From the viewpoint of the Cognitive Scientist, one merit of such procedures over their higher level, and less limited, brethren, is that we can study - and perhaps do something withthe $n$ 's.

\section{The program selector and $\boldsymbol{H}_{2}$}

When we give a computer a problem to work with, we usually give it a program to use. When we give a person a problem, we usually don't provide a program. We expect the person to find one. Our ability to select a good program can play a critical role in our success at dealing with the problem. Choosing programs is what the program selector does.

Suppose, for example, that we are asked to sum the numbers from 1 thru 100 . We can use our regular algorithm, adding the numbers together the long way, or we can realize (as Gauss is supposed to have realized at the age of five) that the numbers to be added can also be thought of as $(1+100)+(2$ $+99)+\ldots+(50+51)=50 \times 101$ and then use the faster multiplying algorithm, that this observation suggests, to compute the result "in our heads".

In some situations we seem to choose the procedure we use, easily and automatically. For example, we quickly call on our digit-recognizing procedure to interpret the $\mathrm{O}$ in 1034, as a digit, and our letter-recognizing procedure to interpret it in BOW, as a letter. In other situations, the choice of a procedure can be difficult and require careful thought.

Program selection seems to be a critical component in several processes. We seem to call on it when we are stuck on a problem and are trying to choose the approach to try next. (In trying to solve the problem, mentioned above, of finding a divisor of $1,000,000$ without zeros, few of us would use 
the exhaustive search procedure. We would search for a more efficient procedure before checking it out.)

We seem to use program selection when we need a new theory (expressed as a program) to replace one that new evidence has just disproved. Or when we daydream. It can be thought of as a process whose input is a partial specification of a program we want and whose output is such a program. Program selection, and the phenomena that depend on it, are not widely studied by Cognitive Scientists (yet). There are undoubtedly many reasons for this but among them are that:

- Program selection tasks are not easily repeated, and thus it is difficult to design experiments that deal with them.

- Program selection is often a component in other processes, and thus not always easily disentangled from the context in which it is embedded.

- Program sclcction may be quite uncomputable, and hence difficult to describe precisely.

There are situations in which program selection is found relatively "pure" and is therefore relatively easy to "see". Its observable properties in some of these situations suggest that, at least at times, it involves more than computing. Consider, for example, the process of computer programming. Programmers generate programs (as outputs) from partial specifications (as inputs). Many people who teach programming know that this process involves more than computation. There is no "Eureka!" moment at which you know your program is correct. But students, at least at first, often do not. Many expect to be able to think carefully (i.e. compute) and come up with a correct program on their first try. They have to be reassured, when they fail to do so, and told that people do not usually (and some don't ever) write a program that works properly the first time. Programming computers, as we do it today, seems to be (at least) a trial and error procedure. If generating programs for computers requires more than computing, we should not be surprised if generating "programs" for other purposes might too.

Another example of a program generation process is the process by which we acquire a skill, such as dribbling a soccer ball, which seems to involve the generation of a program that we "run" when we exercise the skill. That program seems to be acquired by practice, which also looks like a trial and error process. (Some of the practice may go into to developing the required muscles, but some of it is almost certainly used to develop, by trial and error, the program that controls those muscles.)

There is an interesting abstract argument in favor of the claim that program selection requires more than computing. We know that computing cannot increase the amount of information (in the information theoretic sense) and 
usually decreases it. Because the result of a computation is implicit in the program and the data, it cannot, in the information theoretic sense, add to what we know. Thus, for example, $5+7$ contains more information than the result, 12 , since that result could have come from $10+2,4+8,12+0$ or a host of other combinations. Knowing only 12, we cannot reconstruct $5+$ 7. But program selection does increase the amount of information in an information theoretic sense. No finite set of values specifies a predicate uniquely and even all the (infinitely many) values of a computable predicate, $P$, do not specify a unique program, $p$, that computes it. (There are infinitely many such programs, if there are any.) When you know the program, you know more than just the values it computes. Thus we can argue that, since program generation increases information and computation does not, the former cannot be completely reduced to the latter.

To see what kinds of uncomputability might be involved, and to get down to some relatively specific considerations, let's look at the role of program selection in Gold-style induction. Recall that, in the course of finding a correct theory for a predicate, given by its values, we may, from time to timc, find ourselves with a finite set of evidence that contradicts our current theory. When this happens, we need to find a new theory to replace the old. Since theories (in this account) are programs, this calls for program selection. I want to argue that quite general considerations about the nature of the program selection component we use, can have important implications for how successful our procedure for doing induction will be. The more uncomputable the selection process we use can become (up to a point), the more "intelligent" the resulting system can become.

To simplify the discussion, let's assume that the program that our program selection procedurc outputs is always a computing program. Even under this assumption, the procedure that it uses to produce this result need not be a computing procedure. For example, suppose that we are trying to develop a theory of a set that we know to contain $2,4,6$, and 8 . These four pieces of evidence partially specify a program. There are many programs that will meet this (partial) specification and many strategies that we might use to generate such a program. We might use what I will call the "rote strategy" that generates (as its theory) a program that sticks to the facts and does not go beyond them. In this case, such a program would compute YES for 2, 4, 6 and 8 and NO for all other integers. Not only is this program totally computable, but so is the strategy that produced it. This strategy has its uses, but it is not the strategy that we typically use. We are far more likely to theorize, in this case, that the set we are dealing with is the set of all even numbers. What might the process by which we come up with such a theory look like?

One way it might be organized is as follows. It begins with a list of pro- 
grams (theories) that it is willing to consider $\left(T_{1}, T_{2}, T_{3}, \ldots\right)$. Let us call this list its "hypothesis list". The program selection procedure goes through this list and picks the first program on the list that matches the evidence. Obviously, a system based on this strategy can only derive the right hypothesis for a predicate whose program is on its list and so it would make sense to make that list as comprehensive as possible. Let us call the set of all programs (or predicates evaluated by those programs) that such a system can learn inductively, its learning set.

If intelligence is a measure of what such a system can learn, then it should be a measure (of some sort) of the system's learning set. Intelligence tests tend not to measure this (what a system can learn), but rather to measure what a system has already learned. When we consider this situation theoretically, however, we can ask questions about learning sets and about speed of convergence to any given member of that set.

The learning set of an induction method that uses only a totally computable program selection procedure, is quite limited. For example, we know (Kugel, 1975; Nau, 1975) that such a lcarning set can contain only finitely many predicates. That is a serious limitation. Induction methods that use partially computable program selection procedures can have infinite learning sets, but they cannot be "complete" in the sense that they include all possible (totally computable) predicates. There is, however, a slightly more powerful program selection procedure that, when built into an inductive procedure, gives it an hypothesis set that can include all possible (computable) predicates. Here's how such a program selection procedure might work.

We begin with a program that, when run on a computer, generates all the programs in some complete language-a language sufficiently powerful to allow it to express a program to compute any (partially or totally) computable predicate. (All of the popular programming languages have this power.) Using this program-generating program as a subroutine, we construct an $n$-trial procedure that, given a finite set of evidence, will find the first program in the list that matches the evidence. It starts out by computing the position of a rote theory on the list. The result may not be the earliest suitable program on the list, but the earliest program can't come any later. That sets the $n$. It then considers all the programs earlier in the list, trying them out to see if they too match the evidence. Each time it finds a program that does, and that is also earlier in the list than any found so far, it outputs that program. The last program that it outputs is the earliest one in the list that matches the evidence. (Such a procedure can be "complete".) Clearly the program selection procedure is at most an $n$-trial procedure. But the $n$ involved can differ at different places in the induction process. Although we can show that there is no single $n$ that sets a limit on the number of trials needed for all 
inputs, we can, given an input, compute a finite $n$ for that input. We might call such processes "computable trial (or c-trial) processes".

One thing that prevents this program selection process from being totally computable is that some of the programs in the hypothesis list are partial. Their outputs may not be defined for one or more of the pieces of evidence in hand at any given moment. When we try to determine (say) whether the fifth program computes the right output for the input 12 , we may run into a problem if that output is undefined. If it is undefined, and we have an output for 12 in hand, then it is incorrect. But we cannot, generally, tell how long we have to run the fifth program before we can be sure it is undefined and can drop it. If we insist on the earliest program on the list for our theory, then we have to let the process keep running. By letting all the procedures not yet disqualified run on all the finitely many inputs given by the evidence in hand, and picking the earliest one that has been correct for all the evidence at a given point in time, we can be sure that our last theory will be, not only right, but also the earliest in the list. (If we allow the program selection procedure to pick any old "correct" theory, then it can pick a rote theory every time. It is possible that people who feel uncomfortable with the risk of a partially computable theory, might opt for such a strategy. But this move has unfortunate consequences for the scope of their overall inductive procedure.)

The partial hypotheses raise another problem. They cannot be effectively disproved by the evidence. If our current theory is undefined for the input 12 and we have the evidence for that input, we cannot, in general, tell, computably, whether our theory is wrong. And this would keep some partially computable predicates out of our learning set, if we insisted on the total computability of the program selection procedure used in our inductive method.

We could avoid these problems if we could filter the hypothesis list so that it contained only totally computable $\left(\Sigma_{0}\right)$ programs. There are almost certainly situations where this is possible. The process by which we recognize a tune (perhaps represented by a program that generates that tune), from a fragment of that tune that we have just heard, might involve such a list of totally computing programs-with one program to generate each tune we know. Tunes, being finite in length, can be totally computed.

We cannot computably filter out all and only the totally computable programs from a list of all possible programs. But we can do it uncomputably, using a $\Pi_{2}$ filter (see Appendix). A $\Pi_{2}$ program selection procedure, based on such a filter, can lead to an induction method that is complete in the sense that its learning set contains all totally computable predicates.

Without such relatively powerful program selection procedures, we would 
have limited learning sets, which raises some interesting issues. There would be two hasic possibilities. Either different people have different learning sets, in which case one person could learn something that another absolutely could not. Or we all have the same limited learning set and then it might come about that, although we could all learn the same things, we might not be able to learn things known to beings with different learning sets, such as visitors from other planets (if we ever get to see any).

We might have acquired our limited learning sets by evolution. Chomsky (1975), citing Peirce (1903) finds this plausible and Campbell (1974) discusses an epistemology in which the race, rather than the individual, does much of the learning of what is the case in the external world. The ideas of Recursion Theory suggest a mathematical framework for exploring these ideas.

If we have different (incomplete) learning sets, our individual learning sets might represent something like our "intelligence" in the sense that they would represent a limit on what we could learn. Since these sets seem to depend on our program selection procedures, it might make sense to ask whether we could not evaluate our program selection procedures directly, rather than trying to measure (as most intelligence tests do) the set of concepts learned at some particular time. The latter (which Cattell, 1963, called "crystallized intelligence") seems to depend on our personal environment and experiences. Our program selection strategies (which seem to correspond to what Cattell, 1963, called "fluid intelligence") seem to depend on them less.

One merit of this account of intelligence is that it can be made precise and, in terms of it, we can make precise some notions about this concept such as:

- Comparability. Can we compare the intelligence of two arbitrary people? Not necessarily. Consider two systems $A$ and $B$. Let their learning sets be $L(A)$ and $L(B)$. We would like to say that $A$ is more intelligent than $B$ if its learning set, $L(A)$, contains all the predicates (or ideas) that are in the learning set of $B$ (or $L(B)$ ) and then some. Symbolically:

$$
\operatorname{Int}(A)>\operatorname{Int}(B) \Leftrightarrow_{\text {def }} L(A) \text { is properly contained in } L(B) \text {. }
$$

We might then say that the intelligence of two machines $A$ and $B$ is comparable if $\operatorname{Int}(A)>\operatorname{Int}(B)$ or $\operatorname{Int}(B)>\operatorname{Int}(A)$ or $\operatorname{Int}(A)=\operatorname{Int}(B)$. But it is not hard to show that there are situations in which none of these possibilities arises. The set of ideas that $A$ can learn may be different from the set that $B$ can learn without one being included in (or "better") than the other. $A$ 's learning set may be better for one purpose and $B$ 's for another. Knowing something about their learning sets might help us point them in directions in which their learning strategies would be successful, without forcing us to say that one or the other was "better". 
- Measurability. It is possible that human intelligence might have a limited range but, because other assumptions hold, human intelligences are always comparable in the above sense. Let us then say that the intelligence of a set of systems is measurable if there is a computable (effective) procedure for assigning natural numbers to any two systems such the number, $I(A)$, assigned to one system, $A$, was always larger than the number, $I(B)$, assigned to another such system, whenever $\operatorname{Int}(A)>\operatorname{Int}(B)$. Or it might be possible to assign vectors in terms of which the intelligence of systems might be compared multidimensionally. Determining the values of such measures (numbers or vectors) may not be a computable task. That need not mean that nothing about our learning sets can be discovered by computable means, but it should make us cautious.

- The artificial intelligence paradox. The idea that intelligence is a measure of our learning sets, or what we can learn, might shed light on what we might call the artificial intelligence paradox: Once we write a program to do something, we no longer think that doing that thing requires intelligence, and we often say that the human who wrote the program was intelligent rather than the machine that carries it out. This would not be surprising if intelligence were a measure of the programs we can learn from examples, rather than of the programs we can "run".

- Comparing human and machine intelligence. In view of the difficulties we can expect when we try to compare the intelligence of two people, it should not surprise us if it might turn out to be difficult to compare the intelligence of a machine to that of a person. That suggests that it might pay us to be cautious about making such comparisons.

- Learning strategies. There may be more to intelligence than what one can learn. How one learns it may also makes a difference. Two systems with the same learning set can still have different inductive strategies in the sense that, given a set of evidence, they may produce different theories as their "next" guess. The differences between such strategies might be represented as differences in their program selection procedures. Different strategies (in different people) might converge at different rates-and by different routesto the same concepts. Knowing these differences in others might help us to teach them better. Knowing features of our own strategies might help us to learn better. Thinking about the strategies we use might allow us to change them. And it might allow for:

- Learning to learn. A learning strategy is a kind of program that guides the learning process. Suppose that that strategy is itself the object of a learning process (see Figure 6). Then we might talk about how people could adapt their learning processes or "learn to learn". Systems with such an ability can 
Figure 6. Plain learning versus learning to learn.

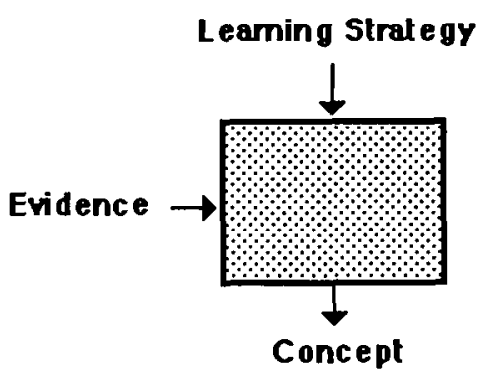

(A) Plain learning

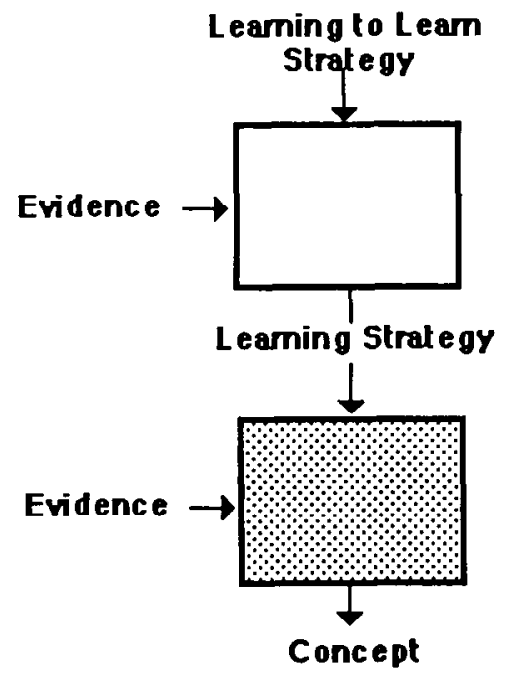

(B) With Learning to Learn

have more comprehensive learning sets than similar systems without this ability. There is some evidence that people can learn to learn and that doing that can help to improve their ability to learn (see for example, Heiman, to appear).

- Learning of paradigms. What Kuhn (1962) has called a "paradigm" may play a role in the intellectual life of the scientist that is not unlike that played by the learning strategy in the intellectual life of the student-it guides him or her in choosing what to look for and do. Paradigms are not theories and are probably not acquired in the same way. This paper's main claim-that thinking may require more than computing — can hardly be a scientific theory in the sense of Popper (1955). How could it possibly be disproved? Why, then, would one adopt it? Perhaps for much the same reason that a student adopts a ncw vicw of the subject being learned or an artist adopts a new way of seeing the world. I have no idea of what those reasons might be, but I suspect that they might reward examination.

Accounts of program selection may play important roles in accounts of the other modules of the mind. In the input processor, program selection may be involved in the process of "seeing as"-the process by which we decide how to look at what we see. In the central processor, it may be involved in 
the process of deciding how to look for a solution of a problem or, more generally, in deciding what to think of next.

\section{Implications for Cognitive Science}

The study of the mind involves two minds-one doing the studying and the other being studied. Some problems of Cognitive Science may arise from the fact that these two minds have the same capabilities. The mind might not, for example, be able to model the mind any more than the world could contain a full-size map of itself and have room for anything else.

The mind could contain a model of itself, if it is a complete $\Sigma_{n}$ or $\Pi_{n}$ procedure for some $n$. Such procedures, like general purpose computing procedures, can model all the procedures in their classes and thus, clearly, themselves. But that may not be enough to make Cognitive Science possible. To do Cognitive Science, the mind also needs to be able to find which of the models of itself that it can construct is the right one. And that may take more power than the mind has.

If it tries to do it by means of Gold-style induction, then it takes a $\Sigma_{n+1}$ or a $\Sigma_{n+2}$ procedure to identify a $\Sigma_{n}$ procedure from its behavior under various assumptions. Thus, even if the mind could model itself and thus, in a sense, handle the theoretical work, it might still not be strong enough to do the scientific work of determining which of its possible models was correct. The study of the mind might, therefore, be impossible, as Fodor (1983) suggests.

Fodor (1983) suggests one way that the scientific study of the mind by the mind might be possible-by breaking it into parts and studying the parts. Dividing the mind into parts could simplify the mind (for the purpose of study) if the mind is a computing system. But it could do so in a particularly important way if the mind is an "uncomputing" system. When we break up an uncomputable procedure into parts, we can decompose it into parts all of which are less uncomputable (and hence presumably simpler to study) than the original (see Appendix).

Trying to predict the behavior of an uncomputable system, even if one knows how it works, raises a special problem because it may be impossible to do that computably. If a mind is a trial and error machine, we might know its inputs and its structure fully and still not be able to compute its result. Thus we might know exactly how such a system worked and not be able to (computably) predict its behavior, if we define that behavior to be its last output. One might say, in such a situation, that the system's result was determined (in the long run) but could not be determined (by a computation). 
This suggests one possible account of free will that does not conflict with determinism.

Ideally, science tries to find computable ways to determine things of interest, but even if there is no computable way to determine certain things about the mind, there may be some things that can be determined by computable means from observations and experiments-and some that cannot. One thing that can almost certainly not be determined effectively from the results of observations and experiments is whether or not the mind can do the uncomputable. Observations and experiments produce finite sets of data and any finite set can be accounted for at any level of the Arithmetic Hierarchy (which includes its lowest level-the level of total computability). Minsky (1967) is right when he warns us that no observation can prove

... that the differences between minds and machines can solve the unsolvable. There is no evidence for this. In fact, there couldn't be. How could you decide whether a given (physical) machine computes an uncomputable (predicate)?

You can't. But that does not mean that one might not choose uncomputable models anyway, much as one might choose the infinite (Turing Machine) models, to which most of Minsky's (1967) book is devoted, over the more finite (Finite Automaton) models, even though one cannot prove, on the basis of finite evidence, that any given physical system is not one of the latter. (Indeed, we know that it has to be. The brain that embodies the mind has to fit inside the skull. Therefore it has to be finite in size and, unless space is infinitely divisible, can have access to only finite amounts of memory. Thus, there is a sense in which it is a finite automaton. But that does not force us to think of it as one.)

There are several reasons for picking one kind of model rather than another-uncomputable, say, rather than computable-even in the absence of any scientific proof that forces us one way or the other. The uncomputable model might produce a simpler theory even though, in some respects, uncomputable models are harder to deal with than computable ones. It might be suggestive, suggesting accounts that a computable account does not. It might produce theories that are smoother-working, or easier to understand, or easier to teach, or that tie more easily to other things that we know.

How one chooses one abstract model of the mind over another is not obvious. For example, consider Chomky's (1956) hierarchy of abstract languages-models that all lie within the computable confines of $\Sigma_{1}$. When Chomsky (1957) argues that the mind cannot be a finite automaton because the English language allows arbitrarily deep embeddings, he is not arguing from observation. We all get lost after a certain depth of embedding. But there are still good reasons for saying that a good account of English requires 
more than a finite automaton. And, in much the same vein, it seems to me that there are good reasons for saying that a good account of the mind requires more than a Turing (or computing) machine.

The suggestion that our descriptions of the mental world might require uncomputable models resembles, in some respects, the suggestion, made several millennia ago, that our descriptions of the physical world might require irrational numbers. Recall that, around the fifth, and early fourth, centuries $\mathrm{BC}$, the Pythagoreans sought to dispell the vagueness and irrationality with which some of their fellow Greeks dealt with nature by claiming that everything could be precisely characterized in terms of numbers. They thought, at first, that the "numbers" were limited to the rational numbers. They showed that many phenomena, ranging from the music of the lute to the eclipses of the sun, could be characterized in "numerical" terms. But then they found magnitudes in the physical world, such as the length of the diagonal of the unit $(1 \times 1)$ square $(=\sqrt{2}$ units), that could not be thus characterized. In the face of this discovery, they seemed to have three basic choices. They could keep their discovery secret, since it weakened their case against those who argued that precise knowledge of the physical world was impossible. They could dismiss it, since the inability to specify $\sqrt{2}$ to more than (say) 100 decimal places is irrelevant to any conceivable practical purpose. Or they could accept it and add the irrational numbers to their kit of conceptual tools. Western civilization ended up choosing the last of these alternatives and expanding its set of conceptual tools. This move made the calculus possible which, in turn, made much of modern science possible.

The uncomputable processes are, to the computable ones, very much as the real numbers are to the rational ones. We cannot finish writing the decimal expansion of certain numbers, such as pi and $\sqrt{2}$, in finite time and we cannot finish evaluating certain trial and error processes in finite time. Both the process by which we derive the irrational numbers from the rational ones and the process by which we derive the uncomputable processes from the computable ones, can be characterized as passing to the limit (Gold, 1965). Adding the results of such processes to the models we allowed ourselves to use in characterizing the physical world, seems to have moved us forward. Adding the results of passing to the limit with computable processes to the set of models we allow ourselves to use in characterizing the cognitive world might move us forward again. Such an analogy does not prove anything. But it is suggestive. 


\section{Conclusion}

I have tried, in this paper, to show how some of the lower parts of the Arithmetic Hierarchy might provide the basis of some models for the uncomputable parts of thinking. What next?

The models I have suggested need to be fleshed out, to be developed in various directions and to have their details filled in so that they can be confronted by the tests of psychological experiment, computer simulation, philosophical argument, linguistic prediction, mathematical analysis, educational practice and, possibly, neurological probing. Alternative uncomputable models might be developed, using the same mathematics differently or using different mathematics.

We are, thus, in much the same situation that Turing (1950) found himself when he finished the paper in which he proposed the use of computable and (although hardly anybody seems to have noticed it) uncomputable models of the mind. "We can," he wrote, "only see a short distance ahead, but we can see plenty there that needs to be done."

\section{Appendix: A sketch of the mathematical ideas}

\section{A1. Introduction}

In this appendix, I want try to give an intuitive account of the main mathematical ideas used in this paper. Although one can, I hope, understand the basic ideas of this paper without understanding the mathematical definitions and proofs that underlie them, an understanding of the mathcmatics can help illuminate and sharpen one's understanding of those ideas. My account will be fairly informal. The reader who wants a fuller, or perhaps just different, account of these arguments might want to consult a text on Recursion Theory such as Rogers (1967) or Shoenfield (1967).

\section{A2. Problems}

I want to develop a fragment of Recursion Theory that can classify problems in terms of the kinds of abstract machinery needed to solve them. Let's start with what I will call "binning problems". A binning problem is a particularly simple kind of problem. We are given a programmable abstract machine, $M$, "seated" behind two bins-one marked YES and the other marked NO. And we are given a classification to make-say to identify all the pictures of males 
in a collection of pictures of people. We are said to have solved the problem, not when we can make that classification properly, but when we can program $M$ to make it properly-when we can produce a program that gets $M$ to put the pictures of human males into the YES bin and the pictures of human females into the NO bin. Usually, we simplify the problem by assuming that all the pictures are clearly focused, that they show the whole person, that pictures of transvestites and other "special" problems are omitted and the like. And we complicate it a bit by assuming that the inputs to be classified are at least potentially infinite so that we cannot look at them all when writing our program.

I will assume that $M$ comes equipped with a transducer, or a device for producing digital representations of the items to be classified in a form that computers can process. And, since there are well-known ways to code such signals as integers, I will consider only binning problems that ask us to classify integers. This may seem rather abstract and limited, but the structure that we develop will turn out to be applicable to a broad variety of problems that deal with things far more concrete, and psychologically interesting, than the integers and involve processes that do not, at first, look like binning at all.

Once you solve a binning problem, you also solve other kinds of problems. For example, if you solve the binning problem for the even numbers, by providing a program that will output YES if the input is an even number and $\mathrm{NO}$ if it is not, you also solve the problems of:

- Evaluating a predicate that is true when the program that solves the binning problem computes YES, and false when it computes NO. For example, the program that solves the "even" problem also computes the predicate, $E$, that is true of all and only the even numbers.

- Determining membership in a set. Thus, for example, our "even" program can be used to determine whether or not a positive integer is in the set, $E$, of even numbers.

I will switch back and forth between problems, predicates and sets, depending on which point of view seems more appropriate.

\section{A3. Computability}

We will say that a binning problem is computable if there exists a computing program that solves it. Let's be (slightly) more specific about what that means. Since we are concerned with idealized computability and want to ignore practical limitations of space, time and the possibility of malfunctions, we will deal with an idealized computer, $M$, that never malfunctions or runs 
out of memory or time. This is an idealization, but it is not very different from the kinds of idealizations with which most mathematical theories used in the sciences (such as physics) begin.

Assume that the programs for $M$ are all written in some fixed programming language (BASIC or Pascal will do). We can enumerate all possible programs for $M$ by listing them in order of increasing length. (In a programming language, there can be only finitely many programs of a given length.) Within a given length, we can list them lexicographically (or "alphabetically"). Given the position of a program in such a list, expressed as an integer, we can compute the program and vice versa-given the program, we can compute its position in the list. We will, therefore, treat programs, as well as inputs, as integers.

A program, $p$, determines the behavior of $M$ for a given input. We say that $M$, with program $p$, "computes a value", $v$, for the input, $i$, if $M_{p}(i)$ (the machine $M$, running under the control of program, $p$, with input, $i$ ) prints $v$ as its first output. As in the body of this paper, we distinguish an output (something $M$ prints) from a result (a selection from all the things it prints that we single out as telling us whether the input belongs in the bin marked YES or NO). When we use $M$ to compute, we take its first output as its result. If that output is neither YES nor NO-that is, if there is no output at all-we say that the result is "undefined". We say that $M_{p}$ totally computes the solution of a binning problem if it computes YES for all integers that belong in the YES bin and NO for all integers that belong in the NO bin. (There are no undefined cases.) And we say that a binning problem (or a set or a predicate) is totally computable if there exists a program that totally computes a solution of the problem (or the set or predicate). Thus the problem of identifying the even numbers is totally computable (as is the set, $E$, of all even numbers or the predicate, $E$, that is true of all and only the even numbers). Another problem that is totally computable is:

The timed halting problem. To find a single program, $s$, (for "stopping") that will place all the halting triples $(p, i, t)$, such that $M_{p}(i)$ halts after $t$ steps, into the YES bin and all others into the NO bin.

To show that this problem is totally computable, we need to show that there is a program, $s$, that totally computes it. That is what is shown by the proof of:

Theorem 1. The Timed Halting Problem is totally computable.

Proof. We prove this theorem, not by actually writing the program, $s$, but by showing that such a program could be written. In doing this, we use a 
thesis and certain lemmas that are used throughout the development of the theory and that should seem plausible to those familiar with computer programming. We begin with the thesis:

The Church-Turing thesis. If we can give an intuitive, step by step, description of how to compute something, we can write a (formal) program for some computing machine that will make it do what we have informally described.

We have not defined what goes into a computing machine and, in a more formal account, we would. But it will do to think of the computing machines as all the computing machines that have been built, idealized to ignore spatial and temporal limitations-as well as limitations of error. Our first lemma is the:

Universal Machine Lemma (Turing, 1936). There is a single abstract machine $M$ that can be programmed to do what any other machine can do.

This says that we can focus our attention on one machine. Any computer on the market will do if we idealize it enough to give it unlimited time and memory space and if we ignore how long it takes to come up with a result. Not only is one machine enough. One program suffices (and all other programs can then be treated as data):

Universal Program Lemma (essentially due to Turing, 1936). For any general purpose computer, $M$, there is a single universal program, $u$, such that $M_{u}(p, i, t)\left(M_{u}\right.$, given $p, i$ and $t$ as input) simulates the behavior of $M_{p}(i)$ for $t$ steps and produces a complete description of $M_{p}(i)$ after $t$ steps of its operation, as output.

This lemma tells us that a program (that is not easy to actually write) exists. If this were a fuller account, I would give more details. I would show how a single program, $u$, would in general, take two integers $p$ and $i$, representing a program and an input to $M_{p}$, as input and simulate $M_{p}(i)$ for $t$ steps. Then I would invoke the Church-Turing Thesis to go from the intuitive description to the claim that the corresponding program could be written.

We need one more general lemma:

The Composition Lemma. The results of combining two computations by composition (applying one to the results of the other) is still a computation.

Now, here's how the required program, $s$, works: Given $p, i$ and $t$, it uses $u$ to produce a description of the state of $M_{p}(i)$ after $t$ steps. $u$ is a computation by our second lemma. $s$ then inspects the description resulting from the first computation to see if it indicates that $M_{p}(i)$ has just halted. If it has, it prints 
YES. Otherwise it prints NO. This second process is also a computation by our version of the Church-Turing Thesis. Therefore, $s$, produced hy composing these computations is also a computation by the Composition Lemma. It is not hard to see that it does what it is supposed to do and that it is totalprinting either YES or NO in all cases. Which proves the theorem.

The Timed Halting Problem is an example of a problem at the very lowest level of uncomputability: none. There exists a computation that handles both the YES's and NO's correctly. Not all problems are totally computable. A well-known example of a problem that is not is:

The Full Halting Problem. To find a program, $h$, that produces YES as its result, for input $(p, i)$, if $M_{p}(i)$ halts (in some number of steps ... not specified, as it was specified in the Timed Halting Problem) and NO if it does not.

The difference between this and the Timed Halting Problem is that, in this problem, we are not given an upper bound on the number of steps of the computation that we have to try before we can say NO. And this is an important difference because we have:

Theorem 2. There can be no totally computable solution to the Full Halting Problem.

Proof. We cannot just say that we have failed to find a program, $h$, that totally computes a solution to this problem, even if we can show that we have looked very hard. There is an infinity of possible solutions and we cannot have looked at all of them. To prove this theorem, we have to show that no such program is possible. In this case, we will show that, no matter what $h$ looks like, it would fail somewhere. And we will do it using a form of argument called a "diagonal argument". The proof goes like this:

Take any totally computing program, $h$, for which it is claimed that it solves the Full Halting Problem. Since $h$ is (by assumption) a totally computing program, we can construct another totally computing program, $d$ (for "diagonalizing"), using $h$, as follows. Given an input $(p, i)$, run $h$. If $h$ computes YES, send $d$ into an infinite loop. If $h$ computes NO, have $d$ stop. By the Church-Turing Thesis and the Composition Lemma, we then know that if $h$ is a totally computing program, then so is $d$. Since we are assuming that $h$ works for all program/input pairs, it should work for the input $(d, d)$ to tell us whether or not $M_{d}(d)$ halts. (Here $d$ is used as both the program and the input. That's OK because both are represented by integers. I'll give a pictorial explanation of this rather odd idea below.) But $h$ won't work on $(d, d)$ because: 
- If $M_{h}(d, d)$ computes YES, then, by the definition of $d, M_{d}(d)$ goes into an infinite loop (and, hence fails to halt). So if $h$ computes YES, it's wrong.

- If $M_{h}(d, d)$ computes NO, then by the definition of $d, M_{d}(d)$ halts and thus $h$ is wrong again.

In other words, $h$ must fail for at least one input: $(d, d)$. But $h$ was any solution to the problem. So any program proposed for $h$ will fail to work properly for at least one input.

The form of this argument is basic to much of Recursion Theory and we will see other versions of it below. It is hard to swallow at first. For one thing, it seems "unfair". We are asking the person who claims that $h$ exists to "go first". We only construct the counterexample after he or she has given a solution $(h)$. The counterexample, $d$, is generally different for each $h$, and it is such a strange and artificial program that one feels that it cannot be very important. Who cares about one exception, and such an odd one at that? But $d$ is only one of an infinity of exceptions that can be constructed in the same general way for any given $h$. What is more, there is no simple way to get rid of all the exceptions without also getting rid of things we want. We cannot get rid of the power needed to carry through this construction without also getting rid of essential features of computations. Not only are diagonal arguments like this one central to the rest of the theory, they also link Recursion Theory to the much older theory of irrational numbers because the existence of irrational numbers can also be proved by a diagonal argument (although this was not done until Cantor did it in the nineteenth century).

To get a better idea of what's behind this form of argument, let's form a two-dimensional picture of the set of all programs that a computing machine, $M$, can evaluate. Write down the program numbers in a list, one to a row. To the right of each program number, write down the values that that program computes for the inputs $1,2,3 \ldots$ in order. Since the program, $h$, that we are representing is totally computable, there is always a YES or a NO computed. The tableau you produce might look like this (with Y for YES and $\mathrm{N}$ for NO) (see Figure 7).

Now consider the diagonal program, $d^{\prime}$, such that $M_{d^{\prime}}(i)$ produces, as its result for the input 1 , the result of program 1 for input 1 , as its result for the input 2, the result of program 2 for input 2 , and so forth. In short, it takes its values from the diagonal of the above tableau, shown in outline, and thus a value from each possible program. (Using our lemmas and our thesis, we can convince ourselves that this program could be written.) Now consider the diagonalizing program, $d$, constructed from $d^{\prime}$, by reversing its outputs: computing NO where the diagonal program computes YES and YES where it 
Figure 7. The diagonal predicate (hollow letters).

inputs (or i)

$\begin{array}{ccccccccc} & 1 & 2 & 3 & 4 & 5 & 6 & 7 & \cdots \\ & 1 & Y & N & Y & Y & N & N & Y \\ \text { programs (or p) } & 2 & N & \mathbb{N} & Y & N & Y & N & N \\ & 3 & N & Y & N & Y & N & N & Y \\ & 4 & Y & Y & Y & N & N & Y & N \\ & 5 & Y & N & N & N & Y & Y & N \\ & 6 & N & Y & Y & Y & N & Y & Y \\ & 7 & Y & N & N & N & Y & Y & \mathbb{N}\end{array}$

computes NO. This program, $d$, cannot be in our list of programs because it differs from each program in that list in at least one result.

$d$ is one of an infinity of programs with this same property. To see how we might produce other "exceptions" for $h$, just think of all the other ways that we might take one value from each program in other ways than along this (main) diagonal. (It just happens that the main diagonal is particularly easy to use.)

Whenever we can arrange a list of programs (or predicates or sets) like this, the "diagonalizing" program (or predicate or set) that we can construct from that list, is not in the list. This gives us the following lemma, stated in terms of predicates, for future use:

The Diagonal Lemma. Whenever there is a method, $L$, for generating a list $\left(P_{1}, P_{2}, P_{3}, \ldots\right)$ of predicates, then the predicate defined by $D(x) \Leftrightarrow$ - $P_{x}(x)$ (where $P_{x}$ is the $x$ th predicate on that list and $\neg$ denotes negation) is not on that list.

\section{A4. Partial computability}

The Full Halting Problem is only "slightly" uncomputable. Part of its solution can be computed. The YES part or:

The Yes Halting Problem. To find a program, $y$, such that $M_{y}(p, i)$ produces the result YES when $M_{p}(i)$ halts (and not otherwise). 
A program, $y$, that solves the Yes Halting Problem differs from the procedure, $h$, that solves the Full Halting Problem in that it need only solve "half" of it. In terms of the binning problem, it has to handle the YES bin properly but need not handle the NO bin fully. (For certain inputs that belong in that bin, it may just "spin its wheels", never deciding, finally, that a given input belongs in the NO bin. But it may not decide, incorrectly, that it belongs in the YES bin.) We say that a binning problem (or predicate or set) is partially computable if there exists a program, $p$, such that $M_{p}$ (inp) computes YES when the solution for inp is YES and not when it is NO.

Theorem 3. The Yes Halting Problem is partially computable.

Proof. Consider the program, $y$, that, given $(p, i)$ simulates the behavior of $M_{p}(i)$ step by step and prints YES if and only if the simulated machine halts. Clearly it computes the right results.

Totally computable problems are also partially computable. (An inspection of the definitions shows that.) But not all partially computable problems are totally computable and the proof of Theorem 2 gives us an exception. Now consider the flip side of the Full Halting Problem or:

The No Halting Problem. To find a program, $n$, that computes NO if and only if $M_{p}(i)$ fails to halt.

Theorem 4. The No Halting Problem is not partially computable (and, hence, not totally computable).

Proof. If it were, we could "dovetail" the procedure, $n$, that computes a solution to the No Halting Problem with the procedure, $y$, that computes a solution to the Yes Halting Problem (which we know to exist, thanks to Theorem 2), to produce a program, $h$, that computes a solution to the Full Halting Problem. But $h$ cannot exist (by Theorem 1) so $n$ can't either. (To dovetail two programs, we run (a simulation of) each, one step at a time in turn, until one of them produces an output.)

A set is said to be "recursively enumerable" if there is a program, $e$, that computably generates (in some order) all and only the members of that set. Any set that is partially computable is also recursively enumerable because we can dovetail the computations for every possible integer and print out that integer when its computation produces a YES. The dovetailing here is a bit more complicated. We simulate a run of the program on the input 1 for one step, then on the inputs 1 and 2 for two steps and so on, simulating its effect 
on the first $n$ integers for $n$ steps. Whenever one on the simulated programs computes a YES, we let the master program print the input that produced that YES. Clearly every integer in the set (for which the program computes YES) gets printed out eventually. The converse is also true: Any set that is recursively enumerable is partially computable. To partially compute it, simply run the enumerating program and, if the input number comes up on the list generated by that program, output YES. Which proves:

Theorem 5. Every partially computable set is recursively enumerable and every recursively enumerable set is partially computable.

Since the set, $N$, of all non-halting program/input pairs is not partially computable, it cannot be recursively enumerable. What is more, there is a computable procedure such that, given any program for which it is claimed that it can list all the non-halting pairs, finds us a non-halting pair not on its list. An uncomputable set for which such an exception-finding program exists is called a "productive sct" (and the program is said to compute a "productive function" for that set). Obviously, a set cannot be both recursively enumerable and productive, but we do have:

Theorem 6. Every productive set contains a recursively enumerable (and hence partially computable) subset.

Proof. Call the productive set $\mathrm{Pr}$ and call its productive function $\mathrm{pr}$. Start with the empty set. Clearly it is recursively enumerable and thus, given an index of it (which is easy to find), the productive function, $\dot{p} r$, will find us a member of the set $\operatorname{Pr}$ that is not in the empty set. Put that number first in the enumeration. Use $p r$ again to find another member of $\operatorname{Pr}$ that is not (yet) in our enumeration. Put it in and repeat, adding each new integer to the enumeration as $p r$ gives it to us and then using an index of the new, partial, enumeration (which is easy to construct since, at each stage, the enumeration produced at that stage is finite) with $p r$ to produce a new member of the list. The result of composing these pieces is a program (by the Composition Lemma) and it recursively enumerates a set, none of whose members is in the given productive set, $\mathrm{Pr}$.

Are there any productive sets? Yes there are, and our set, $N$, of all the non-halting pairs is such a set. The diagonalizing procedure can be used to define a productive function for it. 


\section{A5. The Arithmetic Hierarchy}

The Arithmetic Hierarchy is a hierarchy of predicate classes. Each class, as we move up in this hierarchy, contains predicates that are more uncomputable than any in a lower class. The hierarchy is built up on the same general principles we have used so far and some of the predicates we have (implicitly) been talking about fit into its lower parts. In particular, consider the predicates:

$S$, such that $S(p, i, t)$ is true for all (and only) the triples $(p, i, t)$ such that $M_{p}(i)$ halts in $t$ steps.

$H$, such that $H(p, i)$ is true for all (and only) the pairs $(p, i)$ such that $M_{p}(i)$ halts in some number of steps.

$N$, such that $N(p, i)$ is true of all (and only) the pairs $(p, i)$ such that $M_{p}(i)$ never halts.

$S$ is totally computable (by Theorem 1 ), $H$ is partially computable (by Theorem 3) and $N$ is not computable (by Theorem 4), although its negation $(H)$ is. These three predicates are examples of predicates in the three lowest predicate classes of the Arithmetic Hierarchy $\left(\Sigma_{0}, \Sigma_{1}, \Pi_{1}\right)$. The rest of the Hierarchy adds predicates that are more uncomputable than any in these three classes.

The theorem seems to change the subject because it is based on definability rather than the required machinery, but we will see, in a while, that this can be brought back to machinery in at least some cases. The theorem tells us that, the more quantifiers we use (I'll say what a quantifier is in a moment) to define new predicates in terms of totally computable ones, the more uncomputable the predicates we can define can become.

The predicate $S$ is totally computable so it can be defined in terms of a computable predicate (itself) using no quantifiers. The predicate $H$ can be defined in terms of the totally computable $S$, using one existential quantifier (the existential quantifier ' $(\exists \ldots)$ ' being short for 'There is a ... such that') as follows:

$$
H(p, i) \Leftrightarrow \Leftrightarrow_{\text {def }}(\exists t) S(p, i, t)
$$

(This says that $M_{p}(i)$ halts if and only if there is some step $(t)$ at which it halts. It's not hard to see that that's true.)

Now consider the "going" predicate, $G$, that is the negation of $S: G(p, i, t)$ is truc when $M_{p}(i)$ keeps going after step $t$ and false if it stops. It is easy to see that it, too, is totally computable. (To construct the program that computes it, just switch the YES and NO outputs in the program that computes $S$.) We can define the No Halting Predicate, $N$, in terms of this $G$ predicate, using a single universal quantifier (' $(\forall \ldots)$ ' being short for 'For all ...') as follows: 
$N(p, i) \Leftrightarrow_{\mathrm{def}}(\forall t) G(p, i, t)$

The idea that these examples exemplify can be generalized, and we can show that, the more quantifiers you allow yourself to use in defining new predicates in terms of totally computable ones, the more uncomputable the predicates that you can define will become. Call the set of predicates definable in terms of totally computable predicates using $n$ quantifiers, the first of which is existential, $\Sigma_{n}$. Call the set of predicates definable in terms of totally computable predicates using at most $n$ quantifiers, the first of which is universal, $\Pi_{n}$. Then:

Theorem 7 (the Arithmetic Hierarchy Theorem). For each $n \geq 0$, there are predicates in $\Sigma_{n+1}$ that are not in $\Sigma_{n}$ nor in $\Pi_{n+1}$ and predicates in $\Pi_{n+1}$ that are not in $\Pi_{n}$ or in $\Sigma_{n+1}$ (Kleene, 1943; Mostowski, 1947).

(Figures 2 and 3 in the body of this paper show these relationships.) For example, $\Sigma_{3}$ is the set of all predicates that have definitions of the form:

$$
P(i) \Leftrightarrow_{\mathrm{def}}(\exists x)(\mathrm{Q} y)\left(\mathrm{Q}^{\prime} z\right) R(i, x, y, z)
$$

where $\mathrm{Q}$ and $\mathrm{Q}^{\prime}$ are quantifiers (existential or universal) and $R$ is a totally computable predicate. This class contains predicates not in $\Sigma_{2}$-predicatcs that have definitions of the form:

$$
P(i) \Leftrightarrow{ }_{\mathrm{def}}(\exists x)(\mathrm{Q} y) R(i, x, y)
$$

nor in $\Pi_{3}$-those that have definitions of the form:

$$
P(i) \Leftrightarrow \text { def }(\forall x)(\mathrm{Q} y)\left(\mathrm{Q}^{\prime} z\right) R(i, x, y, z)
$$

What makes this rather irrelevant-seeming theorem relevant to our concerns is that we can relate the machinery needed to define a predicate to the machinery required to evaluate it for some of these classes. For example:

$\Sigma_{0}$ (also known as $\Pi_{0}$ ) is the class of all totally computable predicates. It contains $S .\left(\Sigma_{0}\right.$ represents the class of all effectively decidable predicates-all predicates that can be evaluated by the kinds of computations that a computing center likes.)

$\Sigma_{1}$ is the class of all partially computable predicates. It contains $H$-as well as $S$. ( $\Sigma_{1}$ represents the class of all axiomatizable predicates-in some sense the predicates that might be thought of as the class of all "mathematical", or deductive, predicates.)

$\Pi_{1}$ is the class of all non-halting predicates. It contains $N$-as well as $S$-but not $H$. ( $\Pi_{1}$ represents the class of predicates that are true unless disprovable-which in the sense of Popper, 1955-might be thought of as the "scientific" predicates.) 
That's part of what the lower part of the Arithmetic Hierarchy Theorem "means". Here's why it's true:

Proof. The proof of this theorem is sort of a wholesale use of the diagonal argument. We will take each $\Sigma$ and $\Pi$ class and construct a diagonal predicate, $D^{\prime}$, that takes one argument/value pair from each predicate in (say) $\Sigma_{n}$ but is still in $\Sigma_{n}$. We then take $-D$, the diagonalizing predicate, which (by the Diagonal Lemma) cannot be in $\Sigma_{n}$. $-D$ will turn out to be in both $\Pi_{n}$ and $\Sigma_{n+1}$. So it provides (for each $\Sigma$ class) the required predicate that is in $\Pi_{n}$ and $\Sigma_{n+1}$ but not in $\Sigma_{n}$. (The same argument works for the $\Pi$ classes.)

I'll do the proof using $\Sigma_{3}$ as an example. The proofs for the other classes are similar. (Examples just happen to be easier to explain than the general case.) Before we start, another definition. We say that a predicate $E_{n}(p, i)$ "enumerates" a set of predicates $P_{1}(i), P_{2}(i), \ldots, P_{p}(i), \ldots$ if and only if $E_{n}(p, i) \Leftrightarrow P_{p}(i)$. Thus, if you can evaluate $E_{n}$, then you can evaluate each of the $P_{p}$. We begin with an:

Enumeration Lemma. There is a predicate in $\Sigma_{3}$ that enumerates (and evaluates) all the predicates in $\Sigma_{3}$.

Proof of the Lemma. We can enumerate all the partially computable predicates (in $\Sigma_{1}$ ), using the step-counting predicate $(S)$ with one existential quantifier. In this enumeration, $(\exists t) S(p, i, t)$ represents the $p$ th partially computable predicate. We can use this enumeration to enumerate all the predicates in $\Sigma_{3}$ by enumerating their definitions. Consider the $p$ th predicate in $\Sigma_{3}$, or $P_{p}$. By definition, there is some totally computable predicate $R$ such that:

(1) $P_{p}(i) \Leftrightarrow_{\text {def }}\left(\exists t_{3}\right)\left(\forall t_{2}\right)\left(\exists t_{1}\right) R\left(i, t_{1} t_{2}, t_{3}\right)$

By the definition of $S$ :

(2) $\left(\exists t_{1}\right) R\left(i, t_{1}, t_{2}, t_{3}\right) \Leftrightarrow\left(\exists t_{1}\right) S\left(p, i, t_{1}, t_{2}, t_{3}\right)$.

In logic, as in arithmetic, if you do the same thing to both sides of an equivalence, it remains an equivalence. So by adding similar quantifiers to both sides of (2), we get:

(3) $\left(\exists t_{3}\right)\left(\forall t_{2}\right)\left(\exists t_{1}\right) R\left(i, t_{1}, t_{2}, t_{3}\right) \Leftrightarrow\left(\exists t_{3}\right)\left(\forall t_{2}\right)\left(\exists t_{1}\right) S\left(p, i, t_{1}, t_{2}, t_{3}\right)$

The right hand side of $(3)$ is a predicate with two free (or unquantified) variables. Let's call it $L$ and define it by:

$$
L(p, i) \Leftrightarrow \Leftrightarrow_{\mathrm{def}}\left(\exists t_{3}\right)\left(\forall t_{2}\right)\left(\exists t_{1}\right) S\left(p, i, t_{1}, t_{2}, t_{3}\right)
$$

Since $L(p, i) \Leftrightarrow P_{p}(i), L$ is the enumerating predicate promised in the statement of the lemma.

Now let's turn to the proof of the theorem. $L$ defines a list of one-place predicates $L(1, i), L(2, i), L(3, i), \ldots$ such that $L(n, i)$ is equivalent to the $n$th (one-place) predicate in $\Sigma_{3}$. Notice that, if you can evaluate $L$ (which is one 
of the predicates in $\Sigma_{3}$ ), you can evaluate all the predicates in $\Sigma_{3}$ by plugging in the appropriate value of $p$. We call such predicates complete (or universal) for their classes.

The diagonal predicate for $\Sigma_{3}$ is $D^{\prime}(p) \Leftrightarrow_{\text {def }} L(p, p)$. And the diagonalizing predicate $D$ is:

(4) $D(p) \Leftrightarrow_{\text {def }}-D^{\prime}(p) \Leftrightarrow-L(p, p) \Leftrightarrow-\left(\exists t_{3}\right)\left(\forall t_{2}\right)\left(\exists t_{1}\right) S\left(p, p, t_{1}, t_{2}, t_{3}\right)$.

By the Diagonal lemma, this predicate cannot be in $\Sigma_{3}$. It remains to show that it is in $\Pi_{3}$ and in $\Sigma_{4}$. Again, this involves elementary logical ideas for which the reader, not familiar with such ideas, will have to take my word. Basically, we are going to substitute equivalences. One equivalence is:

(5) $\ldots(\exists x) \ldots \Leftrightarrow \ldots \neg(\forall x)-\ldots$

(Intuitively, what this claims is that saying 'There is an $x$ such that $P(x)^{\prime}$ ' is equivalent to saying 'It is false that, for all $x$, not $P(x)$ '.) Substituting in the right hand side of (4) gives us:

(6) $\quad--\left(\forall t_{3}\right)-\left(\forall t_{2}\right)-\left(\forall t_{1}\right)-S\left(p, p, t_{1}, t_{2}, t_{3}\right)$.

But $-S$ is totally computable too (it's $G$ ) and double negation $(--)$ drops out so that this reduces to:

$$
\left(\forall t_{3}\right)-\left(\forall t_{2}\right)-\left(\forall t_{1}\right) G\left(p, p, t_{1}, t_{2}, t_{3}\right)
$$

with a totally computable $G$. Replacing ' $-\left(\forall t_{2}\right)-$ ' by ' $\left(\exists t_{2}\right)$ ', to which it is equivalent by (5), shows $D$ to be in $\Pi_{3}$. To show it to be in $\Sigma_{4}$ we note that we can always add superfluous quantifiers, and add ' $\left(\forall t_{4}\right)$ ' between the two consecutive negations in (6). We replace $-S$ by $G$, substitute existential for universal quantifiers appropriately, giving us a definition of $P_{p}$ in $\Sigma_{4}$ form.

For readers not familiar with the manipulations of logic, this may seem to be a meaningless game of symbol manipulation, but the basic idea of the proof is nothing more than the diagonal argument used in the earlier proof of Theorem 2. Since we can always quantify variables that don't appear in an expression with no change in meaning we can always add (vacuous) quantifiers. From this it follows that the classes of the Arithmetic Hierarchy at a given level include the lower ones. To be more precise:

Theorem 8. In general, if $m<n$, then $\Sigma_{m}$ contains all the predicates in $\Sigma_{n}$ and $\Pi_{n}$ and, similarly, $\Pi_{m}$ contains all the predicates in $\Pi_{n}$ and in $\Sigma_{m}$.

The intersection of $\Sigma_{n}$ and $\Pi_{n}$ (the class of predicates in both classes) is called $\Delta_{n}$. The lowest levels behave a bit differently than the rest. For example, $\Sigma_{0}, \Pi_{0}$ and $\Delta_{0}$ are all the same class. Therefore, any predicate in any of these classes can be evaluated by the same machinery that can evaluate any of the others and this is why totally computable predicates can be evaluated by both halting and non-halting machines. 


\section{A7. Processes}

Our concern is not so much with predicates as it is with processes that evaluate predicates and with problems that predicates can represent. The classifications of predicates given by the Arithmetic Hierarchy can be carried over to such things. To carry them over, we need ways to represent what quantifiers represent in more "procedural" ways so that we can operate, in these ways, on computations, which we already know how to represent as procedures. This sort of thing is easier to do in the lower parts of the Hierarchy. For example:

$\Sigma_{1}$ : To say that a predicatc, $P$, is in $\Sigma_{1}$ is to say that there is a totally computable $R$ such that $P(x) \Leftrightarrow(\exists y) R(x, y)$. Given an $x$, we can then try out $R(x, 1)$, $R(x, 2), R(x, 3), \ldots$, one at a time. Since $R$ is totally computable, we know we won't get stuck on any of these trials. If $P(x)$ is true, we will eventually find the $y$ that makes it true and be able to print YES. This gives us a "recipe" for turning one process that totally computes $R$ into another that partially computes $P$-for any predicate, $P$, in $\Sigma_{1}$.

$\Pi_{1}$ : To say that $P$ is in $\Pi_{1}$, on the other hand, is to say that there is a computable $R$ such that $P(x) \Leftrightarrow(\forall y) R(x, y)$. Given $x$, we can print YES and then try out all the $R(x, 1), R(x, 2), R(x, 3), \ldots$ one after the other, looking for an exception. Again, the total computability of $R$ assures us that this process will not get "hung up" on a non-halting case. If an exception is found, we erase the YES and leave the result as undefined. This gives us a "recipe" for going from the totally computable procedure for evaluating $R$ to the non-halting procedure for evaluating $P$-for any predicate in $\Pi_{1}$.

$\Sigma_{2}$ : If $P$ is in $\Sigma_{2}$, then there is a totally computable $R$ such that $P(x) \Leftrightarrow$ $(\exists y)(\forall z) R(x, y, z)$. Again, given $x$, we can try out all the $y$ 's one at a time. When we get to a $y$, we print YES, and then we try out all the possible $z$ 's $(z=1, z=2, z=3, \ldots)$. If we find no exception, we keep going, thus never changing the YES for that $y$. If we do find an exception, we print NO, and go on to the next integer for $y$. Clearly $P(x)$ is true if and only if one of the YES's stays forever. This gives us a "recipe" for turning the computable procedure for evaluating $R$ into a trial and error procedure for evaluating $P$ - for any predicate in $\Sigma_{2}$.

$\Pi_{2}$ : If $P$ is in $\Pi_{2}$ then $P(x) \Leftrightarrow(\forall y)(\exists z) R(x, y, z)$. Given $x$, try out each possible $y(y=1, y=2, y=3, \ldots)$. With each new $y$, print YES and try out the possible $z$ 's, holding $y$ fixed. When you find a $z$ such that $R(x, y, z)$, go on to the next $y$. Clearly $P(x)$ is true if and only if this process gets to all the $y$ 's and therefore, prints an infinity of YES's. In these terms, it is easy to see how a $\Pi_{2}$ filter can filter out all possible partially computable processes. In 
effect, it tries out all possible inputs and passes the process only if it halts for all of them.

Going from predicates to processes in this way is a bit ad hoc and there are more general ways. One way is in terms of oracles. An oracle for a predicate, $P$, is a "black box" that a computing machine can use to ask for any values of $P$ that it wishes in the middle of computation. It is easy to see that computing machines with access to a suitable (i.e. complete) oracle, for an uncomputable predicate, can do more than compute.

There are several ways to represent oracles. One way is as a one-way (to the right) infinite tape that contains the graph of the predicate $P$. The graph of $P$ is the sequence whose $n$th value is a 1 if $P(n)$ is true and a 0 if it is false. A computing machine, $M$, can compute all the predicates in a class, $C$, of the Arithmetic Hierarchy if it has access to the oracle of a $C$-complete (or universal) predicate. It is hard to see how to "implement" such oracles in ways that avoid telling the machine, $M$, that uses them, both the YES's and the NO's. That is not a serious problem for our purposes, but the fact that they are actually infinite is. It is hard to think of a way that such oracles could be produced by the mind or, once produced, how they could be made to fit inside our skulls. But this way of representing oracles is useful for mathematical purposes.

An alternative way to represent processes that evaluate uncomputable predicates, is in terms of multi-dimensional time. Adding dimensions to the space available to a computer does not make any noticeable difference in its computing ability. (It may make it faster, but we are ignoring that.) A Turing machine with a one-dimensional tape can compute anything that a Turing machine with an $n$-dimensional tape $(n>1)$ can. But there is a sense in which this is not truc when it comes to time. This is easiest to see if we reformulate the Hierarchy Theorem in terms of the $\Sigma$ and $\Pi$ quantifiers (Kugel, 1977) where ' $(\exists x)^{\prime}$ '... is short for ' $-(\forall x)$ ' (i.e. it stands for 'there is an exception to the following for $x \ldots$..') and ' $(\Pi x)$ ' $\ldots$ is short for ' $(\forall x) \neg$ ' $\ldots$ (i.e. it stands for 'for all $x$, there is nothing but exceptions to ...'). We can then define the classes of the Hierarchy in terms of these quantifiers as follows: $\Sigma_{n}$ is the class of all predicates definable in terms of totally computable predicates, using $n$ $\Sigma$ quantifiers, and $\Pi_{n}$ is the class of all predicates definable in terms of totally computable predicates, using $n \Pi$ quantifiers.

Now, given a predicate in (say) $\Sigma_{n}$, we can think of the process of finding an exception as going off in one orthogonal direction in time until it finds one. Each $\Sigma$ quantifier makes us go off in different dimensions of time. (For further discussion, see Kugel, 1977; Clote, 1986.) It is probably easier to think of ways that this kind of thing might go on, than ways that infinite oracles could be implemented in our finite brains. 


\section{A8. Other objects and other processes}

The characterizations of the Arithmetic Hicrarchy can be carried over to other kinds of mathematical objects. As I noted at the start of this appendix, once you have the machinery in hand to evaluate a predicate, you also have the machinery to solve a problem or to classify things into the set of objects for which a predicate is true (or the 'range' of that predicate). Thus the classification of that Hierarchy can be readily transfered to problems and sets.

Using the Arithmetic Hierarchy to classify functions is slightly less direct. There are various ways that we might identify functions with predicates, but probably the most useful for the concerns of this paper is to identify, with $P$, any selection function for $P$, where a selection function, $f_{P}$, is a function that, for an argument, $x$, has, as value, some integer, $i$, such that $P(i)$. In short, it selects, for an argument, $i$, a value for which $P(i)$ is true. This is basically the idea used in our account of induction in which we accepted any program that computed the full sequence of evidence, even though there are infinitely many such programs if there are any.

The procedures for doing induction do not evaluate ordinary functions of integers. They evaluate functionals, or functions whose arguments are infinite objects such as functions or predicates. A trial and error (or $\Sigma_{2}$ ) functional is evaluated by a process that spits out integers (representing programs) and whose result is the last integer it spits out. Recall that the learning set of such a process is the set of all predicates for which it induces a correct index. It is easy to sec how two such processes might have incomparable learning setssets of predicates for which each machine could successfully do induction but such that neither set was included in the other, as claimed in the body of this paper.

The unmeasurability of such sets is a bit harder to argue for. There are, of course, many ways to "measure" something, but it makes sense to say that whatever way we have, it requires an effective (totally computable) way to assign a measure to a learning set. To make it plausible (but not to prove) that no such measure is possible, we can prove that there is no cffective way, in general, to determine computably whether or not a given predicate is or is not in the learning set of a given procedure. Even if we can "see through" two procedures and determine the index of the predicates they induce, we cannot (in general) tell, by a computation, whether the two are equivalent. (If we could, we could solve the Full Halting Problem. We leave the argument-as mathematical appendixes like this are wont to do-to the reader.) So given an index of a predicate being induced, we cannot tell, computably, even if we can see the theory induced for it, whether or not the predicate will be correctly induced. This suggests that measurement is probably not computable. 
A theorem used in the body of this paper, whose proof we will sketch even more impressionistically than the rest, is about a hierarchy of $n$-trial procedures. Recall that an $n$-trial procedure is a trial and error procedure with an upper bound on the number of times it is allowed to "change its mind". If it changes its mind more than $n$ times, the $n$-trial procedure is said to be undefined. The theorem we used is:

Theorem 9. For any $n$, there are processes that can be evaluated by an $(n+1)$-trial procedure that cannot be evaluated by an $n$-trial procedure.

Outline of proof. Define a predicate that enumerates, for a given $n$, all the $n$-trial predicates, in the style of the enumerating predicate used in the proof of Theorem 2. Then consider the diagonal predicate that takes one value from each predicate in this enumeration. Take the process that $n$-trial evaluates this predicate and diagonalize it as follows. Let the diagonalizing process print a NO before it starts. Now run the diagonalizing process to simulate the evaluation of the diagonal predicate. If this simulated process produces a YES, print a NO and, if it produces a NO print a YES. Keep going, printing the opposite of the diagonal process being simulated. It is not hard to see that the $n+1$ trial process that this defines, produces results that are distinct from each of the results produced by each of the $n$-trial processes for the argument along the diagonal. Therefore, it cannot be an $n$-trial process.

There are other kinds of inputs that the processes discussed in the body of this paper have (other than the infinite graphs of induction) that are not integers. The Arithmetic Hierarchy Theorem classifies predicates on the integers, but cognitive processes seldom deal with the integers. They deal with sentences, images, sounds and many other things. Our classification can be extended to processes with such inputs without changing the basic classifications. One basic rcason for this is the:

Extended Composition Lemma. Using a total computation to preprocess an input, or to postprocess an output, does not change the uncomputability level of a whole process in the Arithmetic Hierarchy.

Thus, the classifications of the Arithmetic Hicrarchy can be used to classify the uncomputability of processes whose inputs are sentences because we can computably map sentences into numbers and recover the sentences from the integers by a computation. We can think of the inputs as being digital versions of pictures (much as an image is digitized on a television screen) because these can be computably mapped into the integers and the image recovered from the integer computably. 
On the other hand, the Composition Lemma fails for uncomputable predicates. Applying an uncomputable process to the results of an uncomputable process can produce a composite that is more uncomputable than either of its components. For example, applying a $\Sigma_{2}$ process to the results of a $\Sigma_{2}$ process can result in a $\Sigma_{4}$ process. This also implies that one can often decompose such a higher level process into several lower level ones. A $\Sigma_{4}$ process can always, for example, be represented as the application of a $\Sigma_{2}$ process to the results of a $\Sigma_{2}$ process. (Again, we leave the proofs of these claims to the reader.)

From the fact that the Arithmetic Hierarchy's classifications of uncomputable processes are not closed under composition, we can show that the kinds of composition used in (say) learning to learn, can extend the learning set of the composite machine. Intuitively, that is not surprising. One would expect a machine that can adapt its learning strategy to be more powerful than one that cannot.

\section{A9. Other machinery}

The uncomputable models of this paper are based on the discrete, deterministic machinery of the digital computer. But models of the parts of thinking that lie beyond computing might, conceivably, be based on different kinds of machinery. Two kinds of machinery that are sometimes proposed are continuous (or analog) machinery and properly random machinery. Although I am not personally impressed by attempts to make such proposals precise, the possibility of models of the uncomputable based on such ideas should not be dismissed out of hand. There are several ways that they might be developed.

I described some ideas for analog models of uncomputable processors in Kugel (1976), but I no longer find any of these convincing. Another such model might be based on a fictional material called Duron, suggested by Martin Gardner (Dewdney, 1985). Duron is properly continuous matter and it appeared in a science-fiction story in which extraterrestrial visitors decide to carry away a written record of all this world's knowledgc. Thcir spacccraft is not big enough to carry it all, so they think of all mankind's writings as a long string of characters. They encode this string by a string of digits and imagine a decimal point on its left. Finally, they take a Duron rod from their spacecraft, notch it so that the ratio between its full length and the length marked off by the notch is exactly the number expressed by that string of digits. They then sail off to their home planet with all of mankind's knowledge represented in that single notch.

If we had a bar of Duron, we might use it to do the uncomputable by taking it and notching it at random. The ratio between the length below the 
notch and the full length of the rod is now some real number between 0 and 1. Represent this number as a binary "decimal" number, represented by a sequence of 0 's and 1's to the right of a binary "decimal" point. Think of this number as encoding a predicate that is true for the argument, $n$, if the $n$th digit in this representation is a 1 , and false otherwise. (It's an oracle.) The resulting predicate is "probably" not computable in the sense that the probability of its being uncomputable is (by the standard definition of such probabilities) 1. This is because the set of all computable predicates is denumerable whereas the set of all predicates (or of all real numbers between 0 and 1 that represent predicates in the manner suggested above) is not. By the standard way of defining probabilities in such cases, the odds of getting a nonmember of a specific denumerable set, when selecting at random from a non-denumerable one, is 1 .

We can then use this binary numeral, represented by a notch, as an oracle for a predicate and evaluate the (almost certainly uncomputable) predicate for any argument by reading off the appropriate digit in its representation. Two problems: First, we don't know what this predicate does. The predicate we can evaluate may be uncomputable and may solve some uncomputable problem, but we don't know what that problem is. Second, we don't have any Duron to work with. Matter, on this planet at any rate, is grainy and cannot be notched with infinite accuracy.

We can get around the second problem by using properly random numbers to generate the sequence of binary digits. We can argue that a sequence of randomly generated 0's and 1's is not computable in the same way that we argued for the uncomputability of the ratio expressed by our randomly notched Duron rod. (Notice that such a sequence could not be produced by the kind of pseudo-random number generator available in many computer languages, because a sequence generated by such a generator is computable.) So, if we had a truly random process, it could give us an uncomputable solution. Unfortunately, I cannot think of any way that we could find out what problem it solves.

Perhaps more viable accounts of how randomness could get us beyond computing might be developed from accounts of probabilistic induction. (See Pitt, 1985, for accounts of such induction.) Or accounts of "uncomputing" machinery might be developed from other ideas.

There are many ideas that might be used to characterize uncomputable processes. But, whatcver ideas we end up using, there are, I think, good reasons for wanting those ideas to be formulated in mathematical terms. Mathematical formulations encourage precision. They allow us to use mathematical results developed by others. They allow us to "divide and conquer" our problems, allowing some to work on the conceptual side while 
others work on the empirical side.

And they may help us to be more open minded. It is one thing to argue over whether computers can or cannot think. One can get quite excited and emotional over that. But it is not easy to get emotional about whether the theory of computability or the theory of uncomputability is a better source of models for the mind. That would be particularly important if it should turn out that good models of the mind can be found in both places.

\section{References}

Aizerman, M.A., Braverman, E.M., \& Rozenoer L.I. (1964). Theoretical foundations of the potential function method in pattern recognition. Automation and Remote Control, 25, 821-837, 1175-1190.

Angluin, D., \& Smith, C.H. (1983). Inductive inference: theory and methods. ACM Computing Survey, 15 , 237-270.

Campbell, D.T. (1974). Evolutionary epistemology. In P.A. Schilpp (Ed.), The philosophy of Karl Popper. La Salle, In Open Court.

Cattell, R.B. (1963). Theory of fluid and crystalized intelligence: a critical experiment. Journal of Educational Psychology, 54, 1-22.

Chomsky, N. (1956). Three models for the description of language. IRE Transactions on Information Theory, IT2, 113-124.

Chomsky, N. (1957). Syntactic structures. The Hague: Mouton \& Co.

Chomsky, N. (1975). Reflections on language. New York: Pantheon.

Clote, P. (1986). A generalization of the limit lemma and clopen games. Journal of Symbolic Logic.

Dekker, J.C.E. (1955). Productive sets. Transactions of the American Mathematical Society, 78, 129-149.

Dewdney, A.K. (1985). Computer recreations. Scientific American, 263, 6, 25.

Dreyfus, H.L. (1979). What computers can't do. (Revised Edition). New York: Harper \& Row.

Epstein, R., Hass, R., \& Kramer, R. (1981). A hierarchy of sets and degrees below 0'. Logic Year 1979-1980. Berlin: Springer-Verlag.

Fodor, J.A. (1983). The modularity of the mind. Cambridge, MA: MIT Press.

Gold, E.M. (1965). Limiting recursion. Journal of Symbolic Logic, 30, $28-48$.

Gold, E.M. (1967). Language identification in the limit. Information Control, 10, 447-474.

Heiman, M. (in press). Learning to learn: a hehavioral approach to improving thinking. In J. Bishop, J. Lochhead, \& D.N. Perkins (Eds.), Thinking. Hillsdale, NJ: Erlbaum.

Hofstadter, D.N. (1982). Metafont, metamathematics and metaphysics. Visible Language, 16, 309-338.

Kirk, G.S., \& Raven, I.F. (1957). The presocratic philosophers. Cambridge: Cambridge University Press.

Kleene, S.C. (1943). Recursive predicates and quantifiers. Transactions of the American Mathematical Society, $53,41-73$.

Kugel, P. (1975). A theorem about automatic programming. SIGART New'sletter, 51, 5-8.

Kugel, P. (1976). Digital to analog conversion (a speculation). SIGACT News, 8, 2, 27-33.

Kugel, P. (1977). Induction, pure and simple. Information Control, 35, 276-336.

Kuhn, T.S. (1962). The structure of scientific revolutions. Chicago, IL: University of Chicago Press.

Leibnitz, G.W. (1956). Philosophical papers and letters. Chicago, IL: University of Chicago Press.

Levine, M.D., \& Melmed, R.D. (1982). The unhappy wanderers: children with attention deficits. Pediatric Clinics of North America, 29, 105-120.

McCarthy, J. (1956). The inversion of functions defined by Turing machines. In C.A. Shannon (Ed.), $A u$ tomata studies. Princeton, NJ: Princeton University Press. 
McCarthy, J. (1980). Circumscription-a form of non-monotonic reasoning. Artificial Intelligence, 13, 27-39.

Miller, G.A. (1956). The magical number seven, plus or minus two: some limits on our capacity for processing information. Psychological Review, 63, 81-97.

Minsky, M.L. (1967). Computation: Finite and infinite machines. Englewood Cliffs, NJ: Prentice-Hall.

Mostowski, A. (1947). On definable sets of positive integers. Fundamenta Mathematica, 34, 81-211.

Myhill, J. (1952). Some philosophical implications of mathematical logic: three classes of ideas. Review of Metaphysics, 6, 165-198.

Nau, D. (1975). A note about the correctness of Kugel's paper. SIGART Newsletter, 54, 3.

Osherson, D.N., Stob, M., \& Weinstein, S. (1986). Systems that learn: An introduction to learning theory for cognitive and computer science. Cambridge, MA: MIT Press.

Peirce, C.S. (1878). Deduction, induction and hypothesis. Popular Science Monthly, 13, 470-482. Reprinted in C. Hartshorne \& P. Weiss (Eds.) (1960). Collected papers of Charles Sanders Peirce. Vol. II (pp. 372-385). Cambridge, MA: Harvard University Press.

Peirce, C.S. (1903). How to Theorize. (The 8th Lowell Lecture of 1903). In C. Hartshorne \& P. Weiss (Eds.) (1960). Collected papers of Charles Sanders Peirce, Vol. V (pp. 413-422). Cambridge, MA: Harvard University Press.

Pinker, S. (1979). Formal models of language learning. Cognition, 7, 217-283.

Pitt, L.B. (1985). Probabilistic inductive inference. Ph.D. Thesis, Computer Science Department, Yale University.

Popper, K.R. (1955). The logic of scientific discovery. New York: Harper \& Row.

Popper, K.R. (1966). The open society and its enemies. Princeton, NJ: Princeton University Press.

Posner, D. (1980). A survey of non-r.e. degrees $\leq 0^{\prime}$. In F.R. Drake \& S.S. Wanier (Eds.), Recursion theory: Its generalisations and applications. Cambridge: Cambridge University Press.

Post, E. (1944). Recursively enumerable sets of positive integers and their decision problems. Bulletin of the American Mathematical Society, 50, 284-316.

Putnam, H. (1965). Trial and error predicates and the solution of a problem of Mostowski. Journal of Symbolic Logic, 20, 49-57.

Reiter, R. (1978). On reasoning by default. Theoretical Issues in Natural Language Processes, 2, 210-218.

Reiter, R. (1980). A logic for default reasoning. Artificial Intelligence, 13, 81-132.

Rosch, E. (1978). Principles of categorization. In E. Rosch \& B.B. Lloyd (Eds.), Cognition and categorization, Hillsdale, NJ: Erlbaum.

Rogers, H. (1967). Thenry of recursive functions and effective computability. McGraw-Hill.

Selfridge, O. (1959). Pandemonium, a paradigm for learning. In D. Blake \& A. Utteley (Eds.). Proceedings Symposium on the Mechanisms of Thought Processes. London: HMSO

Shoenfield, J.R. (1967). Mathematical logic. Reading, MA: Addison-Wesley.

Shoenfield, J.R. (1971). Degrees of uncomputability. Amsterdam: North-Holland.

Simon, H. (1947). Administrative behavior. New York: The Free Press.

Smith, E.E., \& Osherson, D.N. (1984). Conceptual combination with prototype concepts. Cognitive Science, 8, 337-361.

Sternberg, S. (1966). High-speed scanning in human memory. Science, 153, 652-654.

Tversky, A., \& Kahneman, (1983). Extensional versus intuitive reasoning: the conjunction fallacy in probability judgement. Psychological Review, 90 (4), 293-315.

Turing, A.M. (1936). On computable numbers, with an application to the Entscheidungsproblem. Proceedings of the London Mathematical Society, Series 2, 42, 230-265.

Turing, A.M. (1950). Computing machinery and intelligence. Mind, 59, (N.S. 236), 433-460.

Zeigarnik, B. (1927). Über das Behalten von erledigten und unerledigten Handlungen. Psychologische Forschungszeit, 9, 1-85. 
Résumé

Les éléments du raisonnement ne relevant pas du calculable (uncomputable), (s’il en existe), peuvent s'étudier dans l'optique suggérée par Turing (1950) pour l'étude des éléments calculables (computable). On peut rendre compte avec précision des processus cognitifs qui, bien qu'impliquant plus que des calculs, peuvent cependant être modélisés sur ordinateurs. Dans cet article l'auteur propose des modalités pour arriver à ces résultats en utilisant les idées de la théorie mathématique de la Récursion (uncomputability). L'auteur suggère aussi des utilisations pour les modèles que en découlent. (Il est possible au lecteur plus intéressé par les modèles et leurs utilisations que par les mathématiques et les théorèmes de passer rapidement sur la partie mathématique ou d'omettre de la lire.) 